NASA Technical Memorandum 87159

AIAA-86-0312

NASA-TM-87159

19860004753

\title{
Velocity and Temperature \\ Decay Characteristics of \\ Inverted-Profile Jets
}

U. von Glahn, J. Goodykoontz, and C. Wasserbauer

Lewis Research Center

Cleveland, Ohio

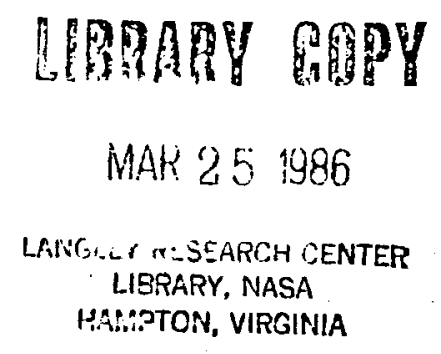

Prepared for the

Twenty-fourth Aerospace Sciences Meeting sponsored by American Institute of Aeronautics and Astronautics Reno, Nevada, January 6-9, 1986

\section{N/Sก}


U. von Glahn, J. Goodykoontz, and C. Wasserbauer

National Aeronautics and Space Administration

Lewis Research Center

Cleveland, Ohio 44135

\section{Abstract}

In order to design efficient, lightweight flap systems for future engine under-the-wing STOL aircraft, the velocity and temperature decay rate of the jet plume must be increased relative to that for single-stream nozzles in order to provide local flap loads and structural temperatures

of within acceptable limits. The jet plume decay

$\underset{\sim}{ }$ rate of dual flow engines can be increased by resorting to inverted-profile velocity and temperature nozzle concepts. In the present paper, the peak axial decay characteristics of model-scale, two-stream inverted-profile nozzle flows are empirically correlated. Also discussed are the radial and spreading characteristics of invertedprofile nozzle flows.

\section{Nomenclature}

A

nozzel area

AR nozzle area ratio, $A_{0} / A_{T}$

b radial half width of outer-stream, ambient side

$b^{\prime}$ radial half width of outer-stream, centerline side

$D_{h} \quad$ hydraulic diameter, $D_{0}-D_{i, 0}$

$\mathrm{O}_{j} \quad$ inner nozzle diameter

$\mathrm{D}_{0} \quad$ outer nozzle diameter

$D_{i, 0} \quad$ inner diameter of outer nozzle

e exponent, defined in text

$F \quad$ correlation factor

$f(x)$ single-stream parameter, $x\left(t_{w} / t_{a}\right)^{0.25} / D_{0} \sqrt{1+M_{w}}$

$M \quad$ jet Mach number

$R$. nozzle radius

$T$ total temperature

$t \quad$ static temperature

$U$ velocity

$x$ axial distance measured from inner nozzle exit plane

\section{Subscripts:}

a ambient

dep departure point

$\begin{array}{ll}i & \text { inner } \\ \text { init } & \text { initial } \\ \mathrm{m} & \text { maximum local } \\ \text { merg } & \text { merged } \\ \mathrm{o} & \text { outer } \\ \mathrm{T} & \text { total } \\ \mathrm{t} & \text { temperature } \\ \mathrm{tran} & \text { transition } \\ \mathrm{v} & \text { velocity } \\ \mathrm{w} & \text { weighted }\end{array}$

\section{Introduction}

For many future aircraft, the control or modification of jet exhaust velocity and/or temperature characteristics, demands increasing consideration. Included among such aircraft are commercial supersonic transports for which the jet exhaust noise must be reduced to acceptable community noise levels. Included also are under-thewing STOL aircraft for which the jet exhaust velocity and temperature must be reduced before impinging on the deflected flap system during STOL operation in order to maintain flap loads and temperatures within acceptable structural limits.

One means of achieving the preceding objectives is through the use of inverted-profile jet exhaust systems. Representative publications include Refs. 1 to 3. In such systems, shown schematically in Fig. 1, core and fan flows can be cross-ducted within the engine nacelle. This procedure provides a relatively thin annulus of hot exhaust flow that decays rapidiy with distance downstream of the nozzle exit plane. Alternatively, as also shown in Fig. 1 , external air could be introduced into the tailpipe by means of an ejector to provide a cold inner flow with the hot engine flow again being redirected to provide a thin annular hot stream surrounding the cold flow. 4 These systems have been shown in the literature to reduce noise levels relative to equal thrust single-stream systems and generally to cause the flow velocity and temperature to decay more rapidly than an equivalent singlestream or conventional dual stream $\left(U_{0} / U_{\mathfrak{j}}<1.0\right)$ exhaust system.

While not considered herein, the decay of inverted-profile velocity/temperature plumes could be enhanced by mechanical or acoustic means as indicated in Ref. 5.

In the present paper, subsonic jet plume velocity and temperature characteristics of inverted-profile coplanar jet plumes are

This paper is declared a work of the U.S. 
correlated for nozzles of three different area ratios. The inner nozzles of these configurations are circular in cross section. The data were obtained over a range of flow rates and temperature levels. Emphasis is placed on the peak axial velocity and temperature decay characteristics, with secondary emphasis being given to the radial and spreading characteristics. Where applicable, the inverted-profile decay data is correlated to the single-stream curves of Ref. 6 .

\section{Apparatus}

\section{Facilities}

Data included herein were obtained primarily from Ref. 7 and from in-house work using the NASALewis dual-flow heated jet facility shown in Fig. 2.

Lockheed facility. ${ }^{2}$ - The nozzle configuration used in this study is shown in Fig. 3(a), together with pertinent dimensions. Note that the configuration is slightly noncoplanar; i.e., the outer nozzle exit plane is upstream of the inner nozzle exit plane. Further details of the facility are given in Ref. 7 .

Lewis facility. - The dual flow heated jet facility shown in Fig. 2 is described in Ref. 8. The nozzles used in the present work are shown in Fig. 3(b) and are the same as those used in Ref. 9. In order to minimize the heat transfer between the two-streams in the nozzle, the outer wall of the inner nozzle was coated with a high temperature ceramic material. The interior of the upstream portion of the inner nozzle supply line was also lined with this insulating material. The following table summarizes the important nozzle dimensions used in the correlation presented later.

\begin{tabular}{|c|c|c|c|c|}
\hline $\begin{array}{l}\text { Refer- } \\
\text { ence }\end{array}$ & $\begin{array}{c}\text { Nozz le } \\
\text { area } \\
\text { ratio, } \\
\text { AR }\end{array}$ & $D_{0}, \mathrm{~cm}$ & $D_{i, 0}, \mathrm{~cm}$ & $D_{i}, \mathrm{~cm}$ \\
\hline 7 & 0.429 & 6.80 & 5.25 & 5.00 \\
9 & $\begin{array}{l}0.653 \\
9\end{array}$ & $\begin{array}{c}17.60 \\
20.758\end{array}$ & $\begin{array}{l}10.81 \\
10.81\end{array}$ & $\begin{array}{l}10.05 \\
10.05\end{array}$ \\
\hline
\end{tabular}

Instrumentation

In general, the instrumentation for the present studies and those of Ref. 7 were similar. Mean flow characteristics were measured using pressure probes and thermocouples mounted in such a manner as to facilitate axial and radial surveys of the jet plume. The measured total and static pressures and the total temperature profiles were used to generate radial profiles of mean velocity, total and static temperature, and Mach number at each axial measurement station.

In addition, to these measurements, instrumentation was provided to measure air flow rates and nozzle pressure and temperature levels. Further details are given in Refs. 7 and 9.

\section{Procedure}

Nozzle pressures and temperatures in the present work and that of Ref. 7 were varied over a range of conditions so that the test matrix included a wide range of jet velocity and temperature ratios. In all cases, steady-state conditions were attained for each flow condition before pressures and temperatures were recorded. Where necessary, upstream total temperatures were corrected for radiation losses and used with the average upstream pressures to calculate an ideal exhaust velocity. Static temperatures were derived from the total temperatures and Mach numbers. Further details of corrections made to the measured data are given in the appropriate references.

\section{General Characteristics of Inverted-Profile Jets}

The basic data used herein were obtained from radial surveys of jet velocity and temperature at several axial stations downstream of the outerstream nozzle exit plane. These data were then used to establish the peak velocity and temperature decay curves. In addition, the individual temperature of each stream was used to provide weighted values of overall temperature and Mach number as will be described iater. It should be noted that the temperatures used are static temperatures, total temperatures show similar trends, albeit at obviously somewhat higher levels.

\section{Radial Profiles}

Typical radial profiles of velocity and static temperature are shown in Fig. 4 for nominal values of $U_{0} / U_{j}=1.5, t_{0} / t_{j}=4.3$ and a nozzle area ratio, AR, of 0.65 . The data indicates that the peak outer-stream velocity decays rapidly with axial distance, $x$, while the peak inner-stream velocity remains substantially constant until both streams are at the same peak local velocity (Fig. 4(a)). Thereafter, the velocity decays in the same manner as a single-stream jet, with the peak velocity occuring at the nozzle centerline. The radial widths of the velocity profiles increase with increasing axial distance, $x$. In general, the outer-stream radial static temperature profiles (Fig. 4(b)) show similar trends to those of the radial velocity profiles, with two differences. For the axial distances shown (up to nearly $130 \mathrm{~cm}$ ), the peak outer-stream temperature remains greater than the peak inner-stream temperature. Also, the inner-stream temperature levels increase with axial distance due to the mixing of the inner-and outer-streams. Although not shown by these data, due to the limits of the measurements available, the temperatures of the two-streams will eventually reach the same level after which the temperature will decay in the same manner as a single-stream jet.

Typical radial velocity and static temperature profiles for nominal values of $U_{0} / U_{j}=1.0$ and $t_{0} / t_{j}=2.38$ are shown in Fig. 5 for $a$ nominal nozzle area ratio of 0.76 . It is evident that the radial velocity profiles are similar to

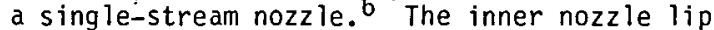
line is clearly evident in the radial velocity profiles near the nozzle exit plane. The radial static temperature profiles and decay show trends similar to those discussed for Fig. $4(b)$ in which both the velocity and temperature profiles were inverted.

Although not shown, for conditions of $t_{0} / t_{i}=1: 0$ and $U_{0} / U_{i}>1.0$, the radial 
velocity profiles show trends similar to those shown in Fig. 4(a) while the static temperature profiles are similar to those for a single-stream nozzle.

\section{Peak Axial Decay}

The peak axial velocity and temperature decay as a function axial distance was obtained from radial profile data shown in Figs. 4 and 5 . Typical peak velocity and static temperature axial decay data are shown in Fig. 6. These data are for nominal values of $U_{0} / U_{j}=1.75, t_{0} / t_{j}=1.75$ and a nominal nozzle area ratio of 0.43 data. ${ }^{7}$ The data are plotted in terms of $U_{m} / U_{0}$ and $\left(t_{m}-t_{a}\right) /\left(t_{0}-t_{a}\right)$ as a function of axial distance from the outer-stream nozzle exit plane, $X$. Also shown for comparison are the respective curves for a single-stream nozzle. ${ }^{6}$ For the two-stream nozzle case shown, the peak velocity and temperature decay trends are similar to each other in that both show a "break" in the decay curve not evident in the single-stream curves. In this respect, the two-stream decay curves are similar to those associated with multielement mixer nozzles included in Ref. 10.

\section{Analysis}

\section{Peak Axial Decay}

In order to correlate the peak axial velocity and temperature decay data, the decay curves shown in Fig. 6 were divided into three segments in a manner similar to that used in Ref. 10 to correlate'multielement nozzles. The three curve segments are illustrated schematically in Fig. 7. The initial mixing or decay region downstream of the nozzle exit plane is associated with the outer-stream flow conditions. At some point along this curve, the rate of decay lessens. The point at which the decay rate characteristics change is designated as the departure point in Fig. 7. The reduced decay rate region is designated as the transition region. Finally, the fully merged flow region is reached at which point the decay is similar to that for a single-stream nozzle. All of these regions are functions of the two-stream nozzle area ratio, AR, and flow conditions.

In the present correlation, empirical equations are developed for each region and establish the departure point. With these equations, decay curves similar to those shown in Fig. 7 can be constructed for a given nozzle configuration and flow conditions. Note that the intersection of the transition curve with the fully merged flow curve is automatically established and need not be specified.

\section{Temperature/Flow Parameters}

Data in Ref. 7 shows that the outer-stream peak static temperature, as well as the total temperature, decreases with axial distance from the nozzle exit plane. However, for the range of conditions included in the study, the peak innerstream temperature remains substantially constant.

Similar trends were obtained in the present study for velocity ratios, $U_{0} / U_{j} \sim 1.5$ and static temperature ratios, $t_{0} / t_{j}$, up to 4.5 , an example is shown in Fig. $8(a)$. For a velocity ratio near 3.5 , however, a marked increase in the peak inner-stream static temperature with axial distance was obtained as shown in Fig. 8(b). This trend is believed due to the increased mixing between the two-streams when the outer-stream is at a significantly higher velocity than that of the inner-stream.

Because of the axial temperature characteristics indicated in Fig. 8, "weighted" fluid dependent parameters were used in the present correlation. These weighted fluid parameters are given by:

$$
t_{w}=\frac{A_{0} t_{0}+A_{i} t_{i}}{A_{T}}
$$

and

$$
M_{W}=\frac{A_{0} M_{0}+A_{j} M_{i}}{A_{T}}
$$

Over the range of conditions included in the available data, the magnitudes of $M_{w}$ and $t_{w}$ differ from the corresponding $M_{0}$ and $t_{0}$ values by up to 10 percent.

\section{Peak Axial Decay Correlation}

\section{Philosophy}

In both the initial and fully merged mixing regions, the correlation equations were formulated so that the two-stream, inverted profile data coincided with the single-stream decay curves given in Ref. 6. Furthermore, the two-stream correlation parameters developed reduce to those for single-stream jets when both $U_{0} / U_{j}$ and. $t_{0} / t_{i}$ are unity.

For the transition region, the correlation equations were formulated to be independent of the single-stream decay curves. However, with some additional complexities, as will be shown later, this correlation can also be reduced to the single-stream decay curves.

In the following sections, the basic data used for developing the correlation equations will be presented with the correlated data.

\section{Basic Data}

In Figs. 9 and 10 are shown the basic data used in the present work in terms of peak velocity and temperature decay as a function of the singlestream correlation parameter, $x\left(t_{w} / t_{a}\right)^{0.25} / D_{0} \sqrt{1+M_{w}}$ taken from Ref. 6 and modified by the weighted Mach number and temperature terms as discussed in the previous section. In general, the invertedprofile data indicate a more rapid decay of the two-stream peak velocity and temperature than that for a single-stream nozzle. Note that for a nominal velocity ratio of 1.0 , the peak velocity decay is independent of the temperature ratio, $t_{0} / t_{j}$ over the range of data available.

\section{Initial Mixing Region}

Peak axial velocity decay. In the initial mixing region, the peak axial velocity decay was correlated in terms of the weighted parameters 
discussed previously and a nozzle geometry parameter is given by:

$$
F_{v, \text { init }}=1+15.25\left(\frac{D_{h}}{D_{0}}\right)^{0.4}\left(1-\frac{D_{h}}{D_{o}}\right)^{3.5}
$$

Thus, the peak axial velocity decay is correlated as follows:

$$
\frac{U_{m}}{U_{0}}-\frac{x}{D_{0} \sqrt{1+M_{w}}}\left(\frac{t_{w}}{t_{a}}\right)^{0.25}\left(F_{v, \text { init }}\right)
$$

The correlation of the data using Eq. (4) together with the single-stream velocity decay curve from Ref. 6 is shown in Fig. 11. It is evident that, in general, good correlation of the data was obtained. Because the two-stream axial velocity decay data coincide with the singlestream decay curve, the relation given by Eq. (4) can be written in equation form, similar to that for single-stream axial velocity decay, as follows:

$\frac{U_{m}}{U_{0}}=\left[1+\left\{\frac{x}{D_{0} \sqrt{1+M_{w}}}\left(\frac{t_{w}}{t_{a}}\right)^{0.25}\left(F_{v, \text { init }}\right)\right\}^{8.0}\right]-0.125$

Peak axial temperature decay. The peak axial static temperature decay was correlated in a manner similar to that used for the velocity decay in the preceding section. For the temperature decay correlation, the nozzle geometry parameter was defined as:

$$
F_{t, \text { init }}=1+6.5\left(\frac{D_{h}}{D_{0}}\right)^{0.167}\left(1-\frac{D_{h}}{D_{0}}\right)^{2.0}
$$

Consequently, the peak two-stream axial static temperature decay is given by:

$$
\left(\frac{t_{m}-t_{a}}{t_{0}-t_{a}}\right)-\frac{x}{D_{0} \sqrt{1+M_{w}}}\left(\frac{t_{w}}{t_{a}}\right)^{0.25}\left(F_{t, \text { init }}\right)
$$

The correlated data using the relationship given by Eq. (7) is shown in Fig. 12 together with the single-stream peak static temperature decay curve from Ref. 6. Again, good correlation of the data was achieved by using Eq. (7).

The relationship given by Eq. (7) can be written in equation form similar to that for single-stream axial static temperature decay as follows:

$\left(\frac{t_{m}-t_{a}}{t_{0}-t_{a}}\right)=\left[1+\left\{\frac{x}{D_{0} \sqrt{1+M_{w}}}\left(\frac{t_{w}}{t_{a}}\right)^{1 / 4}\left(F_{t, \text { init }}\right)\right\}^{8.0}\right]-0.125$

\section{Transition Region}

The velocity and temperature transition regions were correlated in terms of the data departure point from the correlated initial mixing region curve and the slope or decay rate of the transition region curve.

Peak velocity decay. - The peak velocity decay departure point from the initial mixing region to the transition region is given by:

$f(x)_{v, \text { dep }}=\frac{2.7}{\sqrt{D_{h} / 0_{0}}\left(1-\frac{D_{h}}{D_{0}}\right)}\left[1+\frac{0.3}{1+0.2\left(\frac{T_{i}}{T_{0}-T_{i}}\right)}\right]$

The term $f(x)_{v}$, dep is equal to the value of $\left[x\left(t_{w} / t_{a}\right) 0.25\left(F_{v}\right.\right.$, init $\left.)\right] / D_{0} \sqrt{1+M_{w}}$ at the departure point. The transition region velocity decay curve, originating at $f(x)_{v}$, dep is given by:

$$
\left(\frac{U_{m}}{U_{0}}\right)_{\operatorname{tran}}=\left(\frac{U_{m}}{U_{0}}\right)_{\text {dep }}\left(\frac{f(x)_{v, \text { dep }}}{f(x)_{v}}\right)^{0.125}
$$

where $f(x)_{V}$ is measured from the velocity departure point. For $f(x)_{V}$, dep $<4.7$, the exponent in Eq. (10) is given by $1-1 /\left[1+(f(x) y / 6)^{8.0}\right]$ in order to take into consideration the slope of the velocity single-stream decay curve near the nozzle exit plane. For most practical cases, however, the exponent is 0.125 .

Comparisons of the calculated velocity transition region curves with the measured data are shown in Fig. 13. The solid symbols indicate the calculated departure points. It is apparent that the equations represent the data reasonably well.

\section{Peak Temperature Decay}

The peak temperature decay departure point from the initial mixing region to the transition region was analyzed in the same manner as that for the velocity decay. The temperature decay departure point is given by:

$$
f(x)_{t, \text { dep }}=\frac{3.125\left[\left(\frac{t_{0}}{t_{i}}\right)-1\right]}{\frac{D_{h}}{D_{0}}\left(1-\frac{D_{h}}{D_{0}}\right)^{0.67}\left[1+\left(0.26 \frac{D_{0}}{D_{h}}\right)^{6.67}\right]\left(\frac{U_{0}}{U_{i}}-1\right)^{2.5}}
$$

The temperature decay curve, originating at $f(x)_{t, \text { dep }}$, is given by:

$$
\left(\frac{t_{m}-t_{a}}{t_{0}-t_{a}}\right)_{\operatorname{tran}}=\left(\frac{t_{m}-t_{a}}{t_{0}-t_{a}}\right)_{\text {dep }}\left(\frac{f(x) t, \text { dep }}{f(x)_{t}}\right)^{0.25}
$$

Note that the temperature decay exponent is twice that of the velocity decay exponent indicating a more rapid fluid flow temperature mixing in the transition region.

As in the case of the velocity decay, near the nozzle exit, the exponent is less than 0.25 . For the temperature case, the exponent in Eq. (12) for $f(x)_{t, \text { dep }}<4.36$ is given by $1-1 /\left[1+\left(f(x)_{t} / 5\right)^{8}\right]$.

Comparisons of the calculated temperature transition region curves with the measured data are shown in Fig. 14. The equations generally represent the limited available data quite well. 


\section{Correlation to Single-Stream Decay Curves}

Correlation of the preceding transition region velocity and temperature decay data with the respective single-stream decay curve was also determined.

Correlation was achieved by the use of the following relationships:

Velocity. -

$f(x)_{v, \operatorname{tran}}=\left[f(x)_{v, \operatorname{dep}}\left\{\frac{\left(\frac{U_{m}}{U_{0}}\right)_{\text {dep }}}{\left(\frac{U_{m}}{U_{0}}\right)_{\text {tran }}}\right\}^{8.0}\right]^{1-0.2 e^{\prime}}$

where $e^{\prime}$ is the exponent (slope) of the basic single-stream velocity decay curve at the velocity point $\left(U_{m} / U_{0}\right)_{t r a n}$ in question.

Temperature. -

$f(x)_{t, \operatorname{tran}}=\left[f(x)_{t, \operatorname{dep}}\left\{\frac{\left(\frac{t_{m}-t_{a}}{t_{0}-t_{a}}\right)_{\text {dep }}}{\left(\frac{t_{m}-t_{a}}{t_{0}-t_{a}}\right)_{\text {tran }}}\right]_{(14 t)}^{4.0}\right]_{(-0.2 e}^{1-2 e}$

where $e^{\prime}$ is now the exponent (slope) of the single-stream temperature decay curve at the temperature point $\left(t_{m}-t_{a}\right) /\left(t_{0}-t_{a}\right)$ tran in question.

For $f(x)$ transition values greater than about 10 , the value of $e^{\prime}$ for both velocity and temperature is 1.0; hence the exponent in Eqs. (13) and (14) is 0.8. With $f(x)$ values less than 10, the $e^{\prime}$ becomes less than 1.0; consequently, the exponent approaches 1.0 as $f(x)$ is reduced and $U_{m} / U_{0}$ and $\left(t_{m}-t_{a}\right) /$ $\left(t_{0}-t_{a}\right)$ values approach 1.0

In general, the added complication of correlating the transition region decay data with the single-stream decay curve is not considered necessary.

\section{Fully Merged Flow Region}

The final segment of the peak two-stream axial decay curve is designated as the fully merged flow region.

Peak axial velocity decay. - The peak axial velocity decay in the fully merged flow region requires not only. a nozzle geometry parameter, as in the initial mixing region, but a parameter involving the nozzle velocity and temperature ratios. These parameters are given by the following equation:

$F_{v, \text { merg }}=\left[1+5.5\left(\frac{D_{h}}{D_{0}}\right)\left(1-\frac{D_{h}}{D_{0}}\right)^{4}\right]\left(\frac{U_{0} t_{i}}{U_{i} t_{0}}\right)^{0.167}$
The peak axial velocity decay is then correlated by:

$$
\frac{U_{m}}{U_{0}} \sim \frac{x}{D_{0} \sqrt{1+M_{w}}}\left(\frac{t_{w}}{t_{a}}\right)^{0.25}\left(F_{v, \text { merg }}\right)
$$

The correlation of the velocity decay data in the fully merged flow region using Eq. (16) together with the single-stream decay curve from Ref. 6 is shown in Fig. 15. In general, reasonably good correlation of the data has been achieved by the present correlation means.

The relationship in Eq. (14) can be written in equation form as:

$\frac{U_{m}}{U_{0}}=\left[1+\left\{\frac{x}{6 D_{0} \sqrt{1+M_{w}}}\left(\frac{t_{w}}{t_{a}}\right)^{0.25}\left(F_{v, \text { merg }}\right)\right\}^{8.0}\right]-0.125$

Peak axial temperature decay. - The peak static temperature decay was correlated in a manner similar to that used for the velocity decay. However, based on the data of Ref. 7, the temperature decay correlation indicate the inverse function of the $\left(U_{0} t_{j} / U_{j} / t_{0}\right)$ parameter used for the velocity decay correlation. Thus, the temperature decay correlation parameter is given by:

$F_{t, \operatorname{merg}}=\left[1+2.2\left(\frac{D_{h}}{D_{0}}\right)^{0.25}\left(1-\frac{D_{h}}{D_{0}}\right)^{3.0}\right]\left(\frac{U_{i} t_{o}}{U_{0} t_{i}}\right)^{0.167}$

The peak axial temperature decay is then correlated by the following relationship:

$$
\left(\frac{t_{m}-t_{a}}{t_{0}-t_{a}}\right) \sim \frac{x}{D_{0} \sqrt{1+M_{w}}}\left(\frac{t_{w}}{t_{a}}\right)^{0.25}\left(F_{t, \text { merg }}\right)
$$

The correlated data using Eq. (19) is shown in Fig. 16 together with the single-stream temperature decay curve. In general, good agreement of the data, using the relationships shown, with the reference curve was obtained.

The relationship shown by Eq. (19) can be expressed in equation form as follows:

$$
\left(\frac{t_{m}-t_{a}}{t_{0}-t_{a}}\right)=\left[1+\left\{\frac{x}{D_{0} \sqrt{1+M_{w}}}\left(\frac{t_{w}}{t_{a}}\right)^{0.25}\left(F_{t, \text { merg }}\right)\right\}^{8.0}\right]^{-0.125}
$$

\section{Radial Decay Characteristics}

For two-stream inverted profile jets, the ambient side of the outer-stream is subjected to the same radial decay phenomenon as that associated with the boundary of a single jet. On the inner side of the outer-stream, however, the reference velocity/temperature condition is no longer the ambient condition but rather that of the inner-stream. Once the two flows (velocity and/or temperature) are fully merged, the maximum (reference) flow values are those at the nozzle centerline and the radial decay is similar to that associated with single-stream nozzles. 
Pertinent variables needed for correlating the radial velocity and temperature profiles of two-stream inverted-profile jet flows are shown in Figs. 17 and 18 for the initial mixing region and the fully merged flow region, respectively. In the following sections, typical velocity and temperature radial decay rates associated with two-stream inverted-profile jets are compared with those for single-stream jets.

\section{Radial Velocity Profiles}

On the ambient side of the outer-stream, the radial velocity decay is given by $\mathrm{U} / \mathrm{U}_{\mathrm{m}}$. In Fig. 19(a) to (c), are shown typical correlated two-stream velocity profile decay data for the ambient side in terms of $U / U_{m}$ as a function of $R / b_{v} o^{*}$ Also shown in the figure is the singlestream radial velocity decay curve taken from Ref. 6. It is apparent that the ambient side of the two-stream radial decay is correlated in the same manner as that for a single-stream flow. The correlation applies to both the initial mixing and fully merged flow regions.

On the inner side of the outer-stream flow, the radial velocity decay is given by $U_{m}-U_{m}$ (Fig. 17(a)). Typical correlated velocity profile data for the inner portion of the outer-stream flow as a function of $R / b^{\prime} v, o$ in Fig. 19(d) together with the single-stream decay curve. Good agreement is again noted between the inverted profile data and single-stream curve.

\section{Radial Temperature Profiles}

On the ambient side of the outer-stream, the radial temperature decay is given by $\left(t-t_{a}\right) /$ $\left(t_{m}-t_{a}\right)$. The variation of this parameter with $R / b_{t}$, is shown in Fig. 20(a) to (c), for several flow'conditions. Also shown in the figure for comparison is the single-stream curve taken from Ref. 6. Reasonable agreement between the twostream and single-stream radial temperature decay rates is evident, as was the case for the radial velocity decay rates.

On the inner-stream portion of the outerstream, the radial temperature decay is given by $\left(t-t_{m, j}\right) /\left(t_{m}-t_{m, j}\right)$ (see Fig. 17(b)). The variation of this parameter with $R / b^{\prime} t$ is shown in Fig. 20(d) together with the reference single-stream curve. Good agreement between the two-stream and single-stream decay rates was again obtained.

\section{Radial Width}

The radial width characteristics of invertedprofile jets herein are presented in terms of the half-width velocity and temperature values as a function of axial distance from the nozzle exit plane. These values are nondimensionalized by the outer nozzle radius, $R_{0}$.

Velocity

Representative data of the radial velocity width characteristics as a function of axial distance, $X / R_{0}$ are shown in Figs. 21 and 22 . Also shown in the figure is the radial velocity width curve for a single-stream conical nozzle taken from Ref. 6. For conditions and nozzle area ratios shown, the radial velocity width on the ambient side of the outer-stream relative to the outer nozzle lip line is nearly constant over the range of $X / R_{0}$ values for the data. In general, the radial velocity width decreased somewhat relative to the lip line with an increase in velocity ratio. An increase in temperature ratio also generally had a tendency to cause the radial velocity width to decrease slightly, although this trend may be fortuitous in the data.

On the inner side of the outer-stream, the radial velocity width decreased significantly with an increase in velocity ratio, $U_{0} / U_{i}$, as shown in Fig. 22. An increase in temperature ratio, $t_{0} / t_{i}$, generally appeared to have little effect on the radial velocity extent. The data shown in Fig. 22(b) indicate a significant reduction of the radial velocity width at the largest $X / R_{0, i}$ value measured.

\section{Temperature}

Representative data of the temperature radial width characteristics as a function of axial distance, $X / R_{0}$, are shown in Figs. 23 and 24 and compared with those of a single-stream jet. The radial width for the two-stream nozzle was always less than that of a single-stream conical nozzle. In general, the two-stream jet width decreased with an increase in velocity ratio, $U_{0} / U_{j}$, and also with an increase in temperature ratio, $t_{0} / t_{i}$. These effects were more pronounced for the smaller area ratio nozzle (Fig. 23(a)) than for the larger area ratio nozzle (Fig. 23(b)).

In Fig. 24 are shown the radial temperature width characteristics on the inner side of the outer-stream. In general, the temperature characteristics are similar to the velocity width characteristics on the inner side of the outerstream. For the most part, the temperature width extends rapidly toward the inner-stream centerline, as might be expected.

An increase in velocity ratio, $U_{0} / U_{i}$, generally tends to increase the inward spreading rate of the temperature, while an increase in temperature ratio, $t_{0} / t_{i}$, tends to retard the spreading rate. In general, these effects are more pronounced for the smaller area ratio nozzle of the two shown in the figure.

\section{Conclusions}

On the basis of the present correlation concerned with the velocity and thermal characteristics of two-stream inverted-profile jet plumes, the following conclusions are made:

1. Two-stream inverted-profile jet peak axial velocity and temperature decay measurements were empirically correlated by suitable geometry and flow parameters that in their limits reduce to previously published single-stream jet correlation equations.

2. The two-stream, inverted-profile peak velocity and temperature decay curves consist of three distinct parts similar to those associated with jet noise suppressor nozzles; namely:

(a) Initial mixing region in which the outer-stream properties dominate the axial veloc$i$ ty and temperature decay characteristics. 
(b) A fully merged flow region in which the peak velocities and temperatures occur at the nozzle centerline.

(c) A transition region between the initial and fully merged flow regions.

3. Over the range of variables included in the present work, the peak axial velocity decay with equal stream velocities was independent of outer-to-inner-stream temperature ratio.

4. Two-stream, inverted-profile radial velocity and temperature decay data were correlated by similar techniques to those used for conventional single-stream jets.

5. Inverted-profile spreading rates are less than those for single-stream jets having equal overall nozzle diameters.

\section{References}

1. Knott, P.R., Blozy, J.T., and Staid, P.S., "Acoustic and Aerodynamic Performance Investigation of Inverted Velocity Profile Coannular Plug Nozzles," NASA CR-3149, 1981.

2. Larson, R.S., Nelson, D.P., and Stevens, B.S.: "Aerodynamic and Acoustic Investigation of Inverted Velocity Profile Coannular Exhaust Nozzle Models and Development of Aerodynamic and Acoustic Prediction Procedures," NASA CR-3168, 1979.

3. Vdoviak, J.W., Knott, P.R., and Ebacker, J.J., "Aerodynamic/Acoustic Performance of YJ 101/Doudle Bypass VCE With Coannular Plug Nozzle," General Electric Co., Cincinnati, OH, R80AEG369, Jan. 1981. (NASA CR-159869).
4. Klees, G.W. and Wright, C.P., "Internally Ventilated Noise Suppressor with Large Plug Nozz le," U. S. Patent $4,501,393$, assigned to The Boeing Company, Feb. 26, 1985.

5. von Glahn, U.H., "Preliminary Analys is of Tone-Excited Two-Stream Jet Velocity Decay," NASA TM-86951, 1985.

6. von Glahn, U.H., "On Some Flow Characteristics of Conventional and Excited Jets," AIAA Paper 84-0532, Jan. 1984.

7. Tanna, H.K., Tester, B.J. and Lan, J.C., "The Noise and Flow Characteristics of Inverted-Profile Coannular Jets," Lockheed-Georgi a Co., Marietta, GA, Feb. 1979. (NASA CR-158995).

8. von Glahn, U.H., Goodykoontz, J. and Wasserbauer, C., "Velocity and Temperature Characteristics of Two-Stream, Coplaner Jet Exhaust Plumes," AIAA Paper 84-2205, 1984.

9. Goodykoontz, J.H. and Stone, J.R., "Experimental Study of Coaxial Nozzle Exhaust Noise," AIAA Paper 79-0631, Mar. 1979.

10. Groesbeck, D.E., Huff, R.G. and von Glahn, U.H., "Comparison of Jet Mach Number Decay Data With a Correlation and Jet Spreading Contours for a Large Variety of Nozzles," NASA TN-D-8423, 1977. 


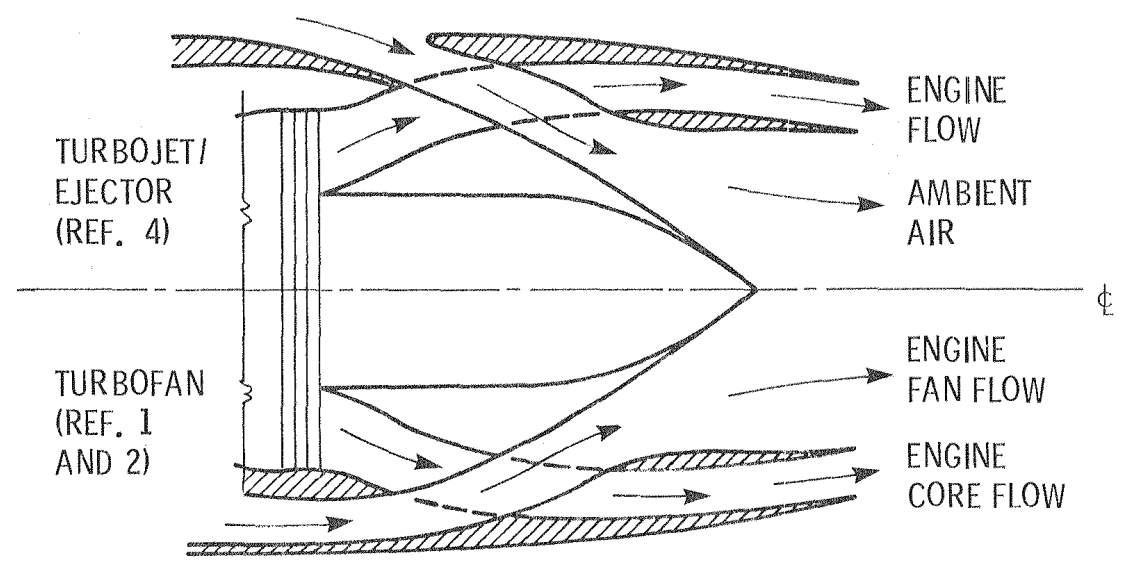

Figure 1. - Schematic of inverted-profile nozzle concepts.

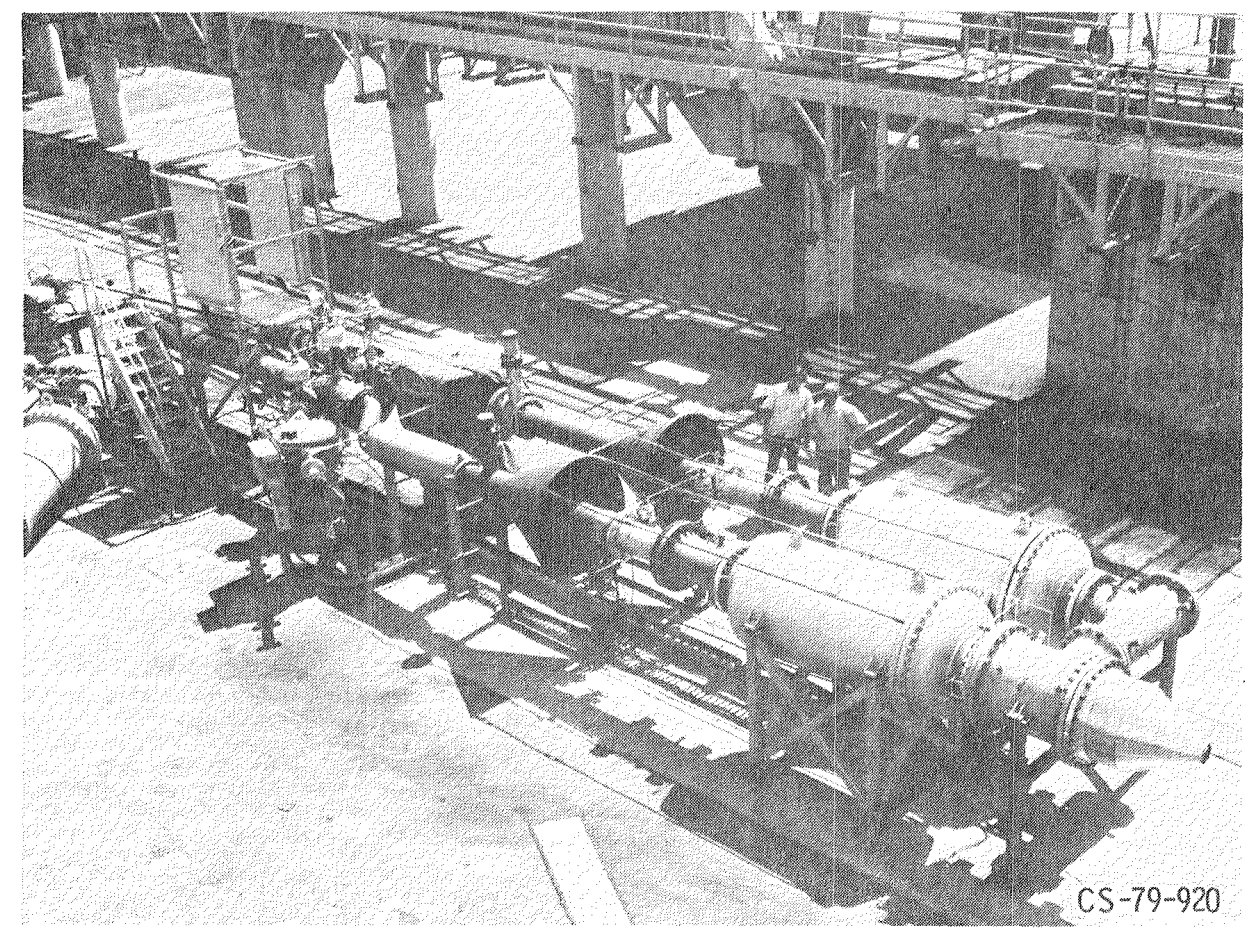

Figure 2。 NASA-Lewis Dual-Stream heated jet facility. 


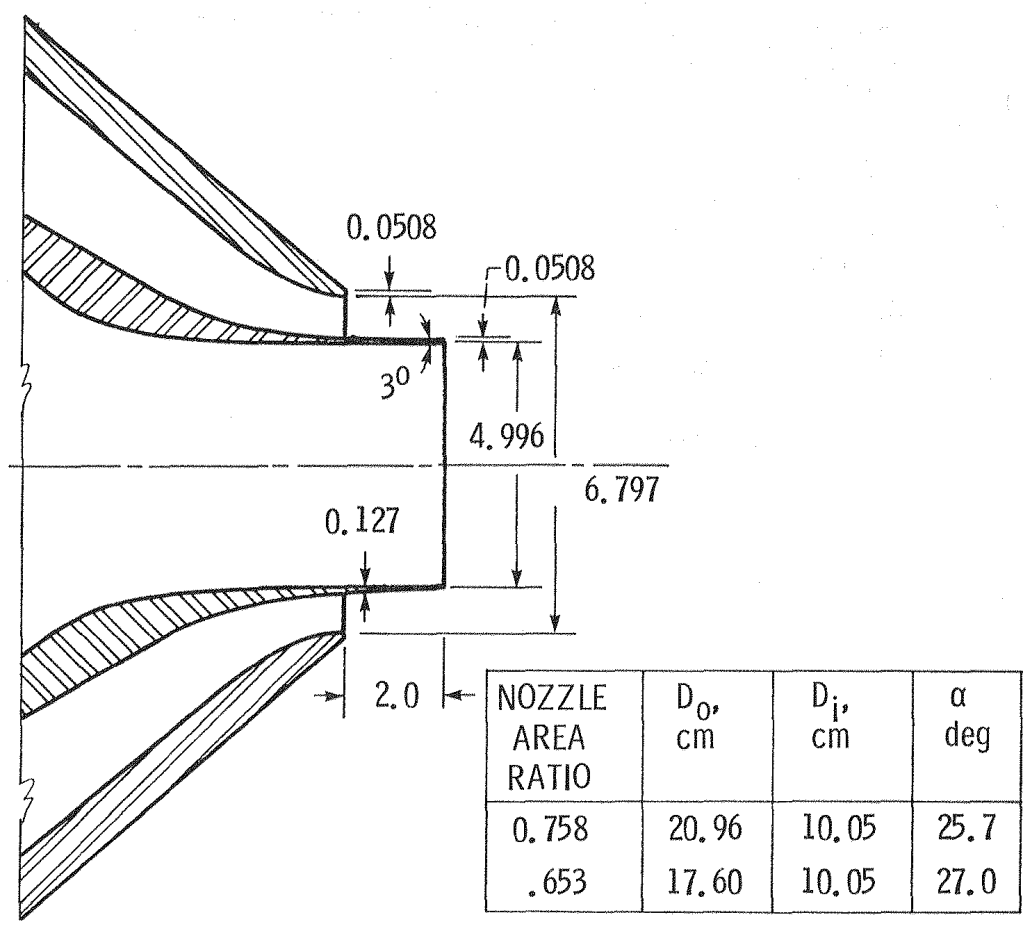

(a) Two-stream nozzle, ref. 6; nozzle area ratio, $A R, 0.4288$.

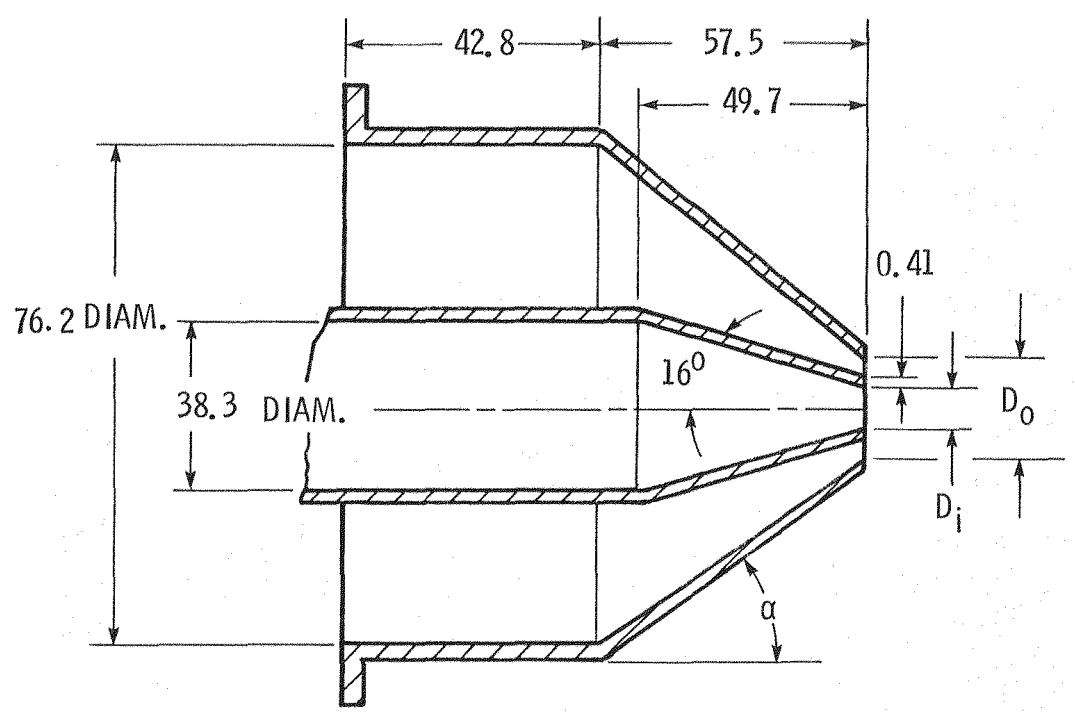

(b) Coplanar two-stream nozzle, ref. 9.

Figure 3. - Schematic of two-stream nozzles. All dimensions in centimeters. 


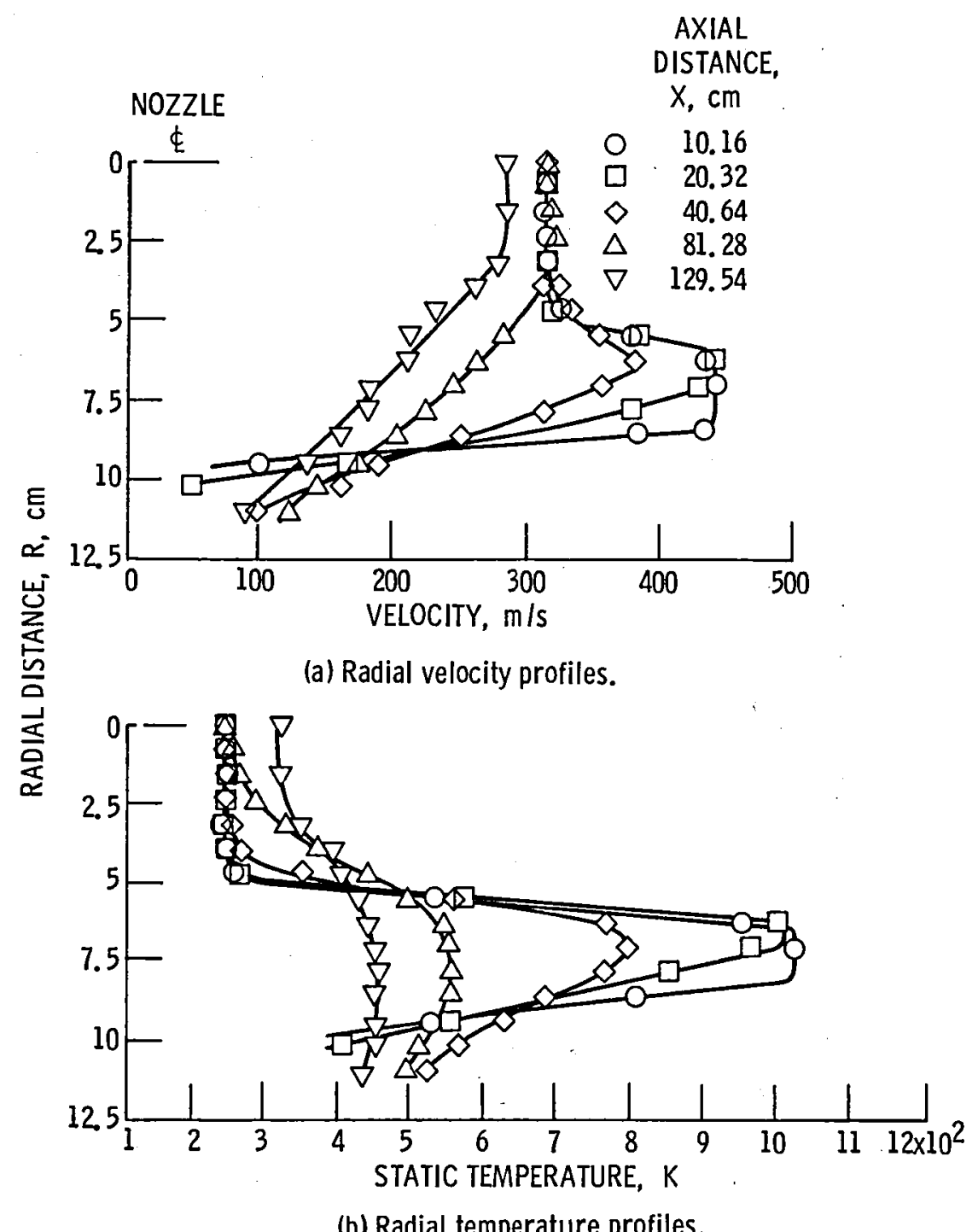

Figure 4. - Typical radial velocity/temperature profiles for both $u_{0} / u_{i}$ and $t_{0} / t_{i}>1.0 . U_{0} / U_{j}, 1.5 ; t_{0} / t_{j}, 4.3 ; A R, 0.65$. 


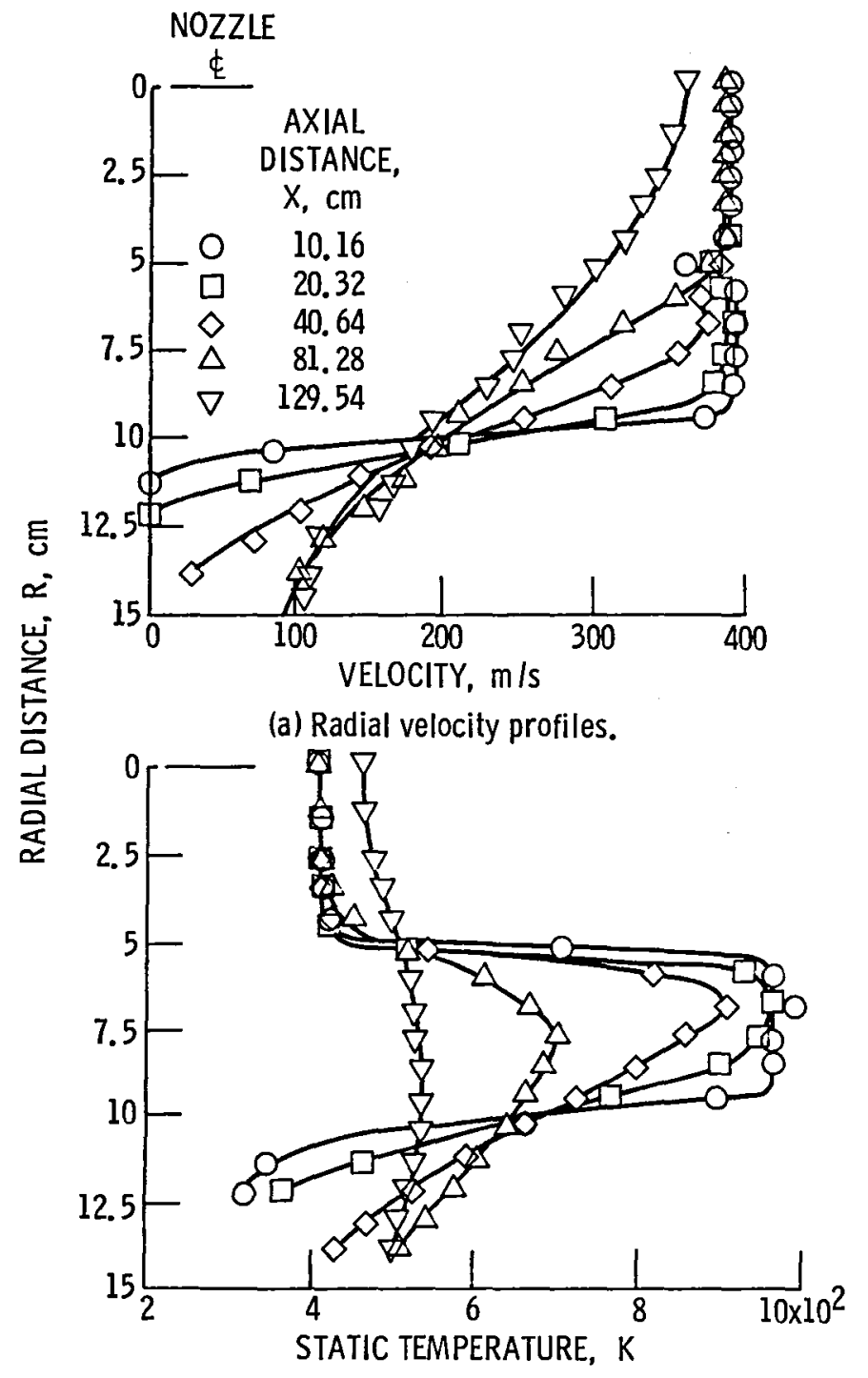

(b) Radial temperature profiles.

Figure 5. - Typical radial velocity/temperature profiles for $U_{0} / U_{i}=1$ and $t_{0} / t_{j}>1.0 . t_{0} / t_{j}, 2.38$; $A R, 0.76$. 


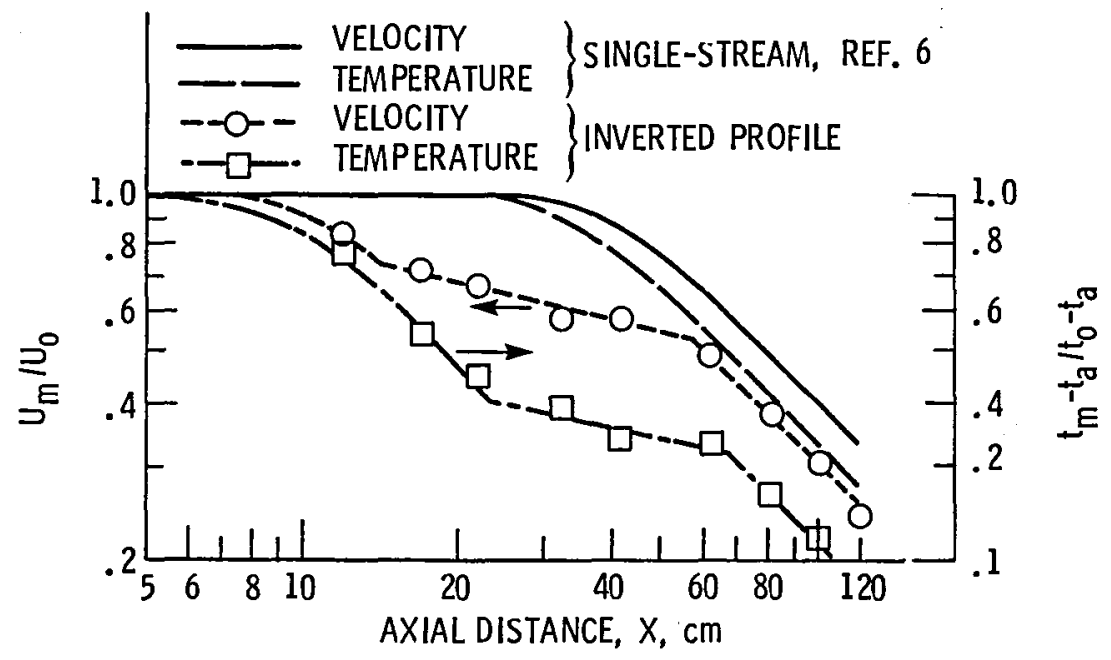

Figure 6. - Representative two-stream inverted-profile velocity /temperature decay data. Nominal nozzle AR, $0.43 ; U_{0} / U_{i} \sim 1.75 ; t_{0} / t_{i} \sim 1.75$; ref. 7 data.

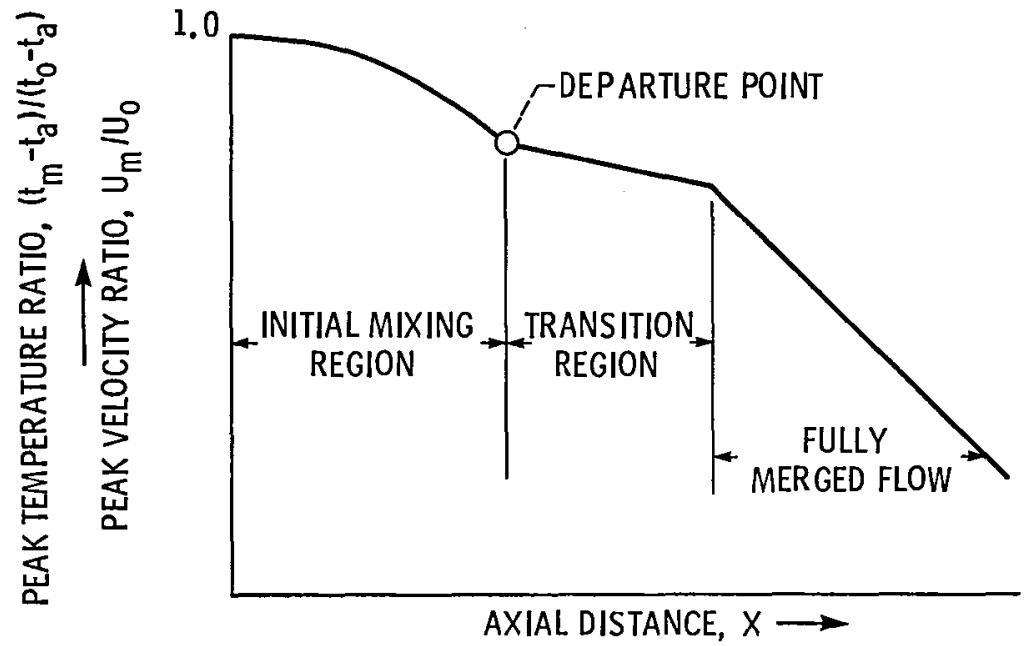

Figure 7. - Schematic of inverted-profile velocity/ temperature decay curve segments. 


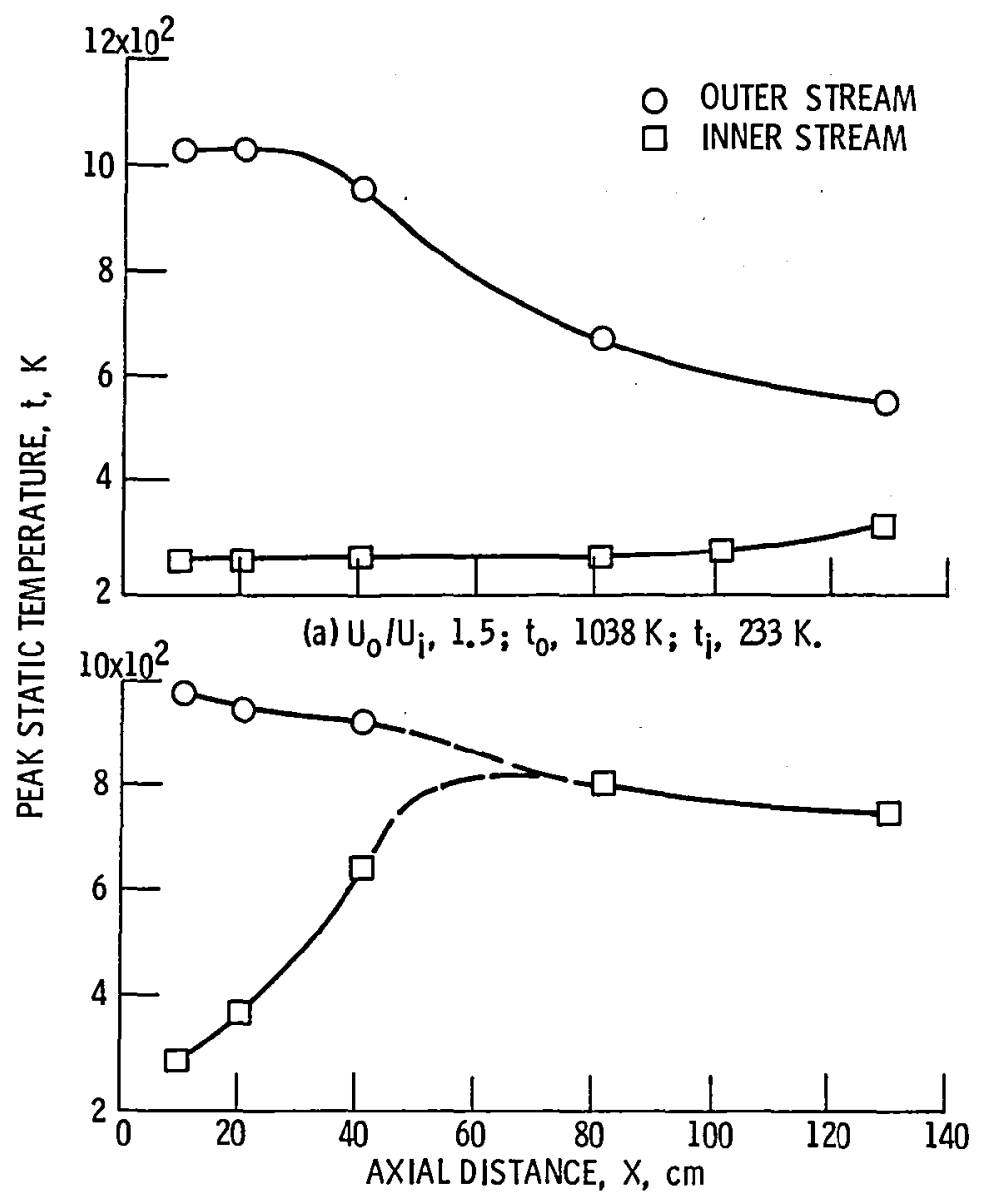

(b) $U_{0} / U_{i}, 3.5 ; t_{0}, 968 K_{;} t_{i}, 274 K$.

Figure 8. - Effect of velocity ratio, $U_{0} / U_{i}$, on peak inner stream temperature. Nominal AR, 0.76 . 


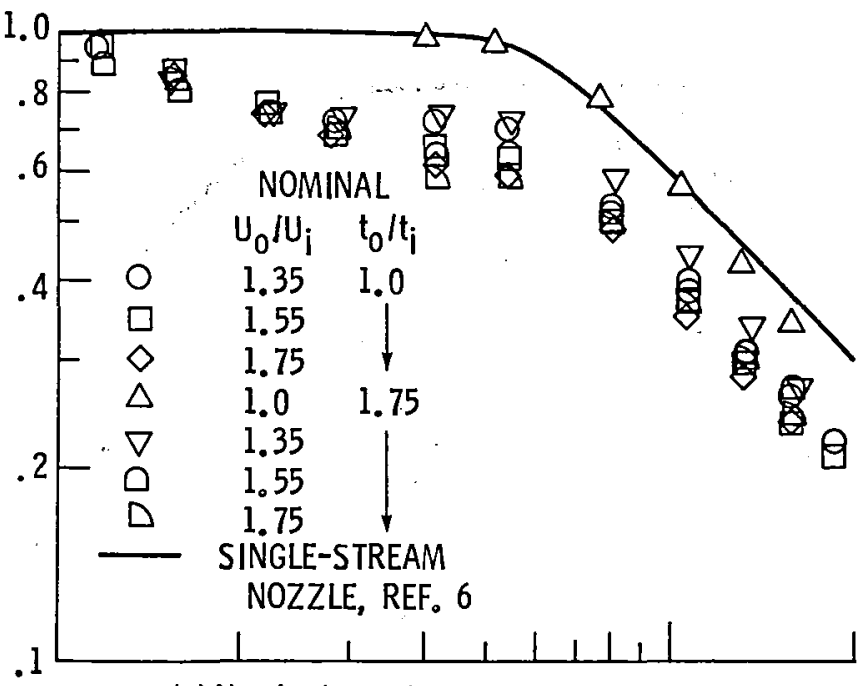

(a) Nominal nozzle AR, 0.43; ref. 7 data.

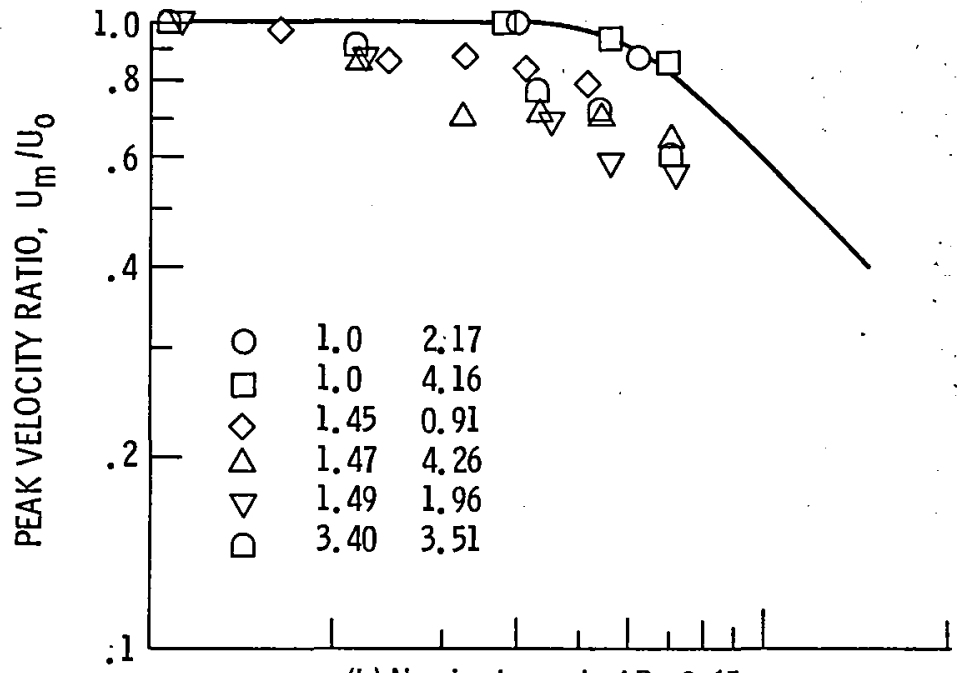

(b) Nominal nozzle AR, 0.65 .

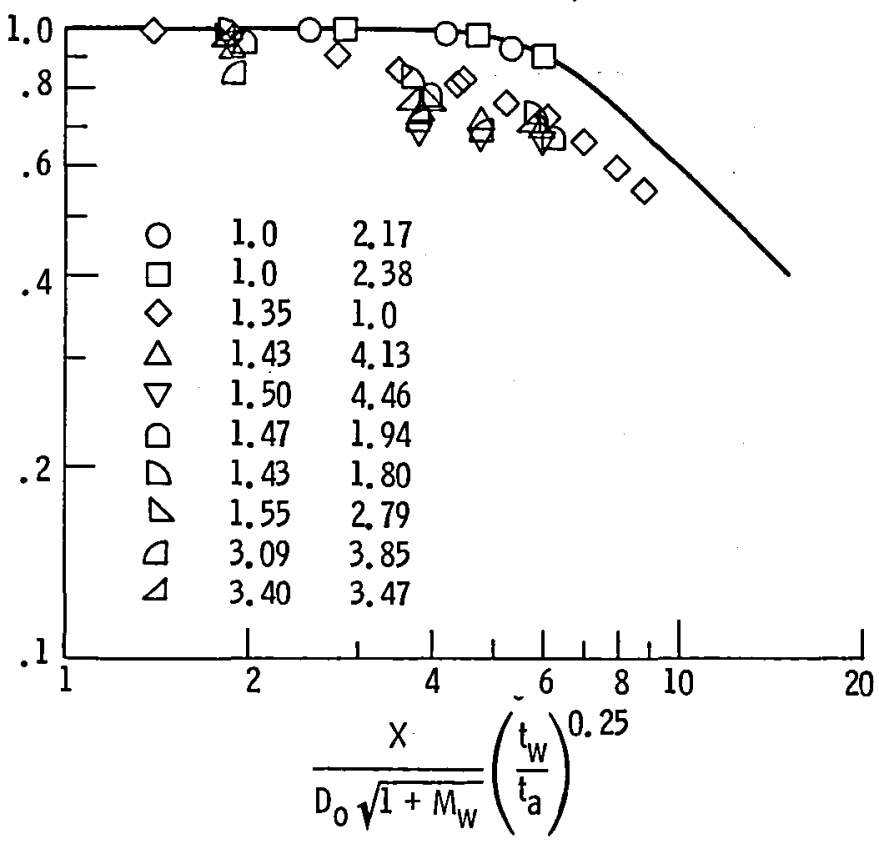

(c) Nominal nozzle AR, 0.76 .

Figure 9. - Peak two-stream inverted-profile velocity decay data. 


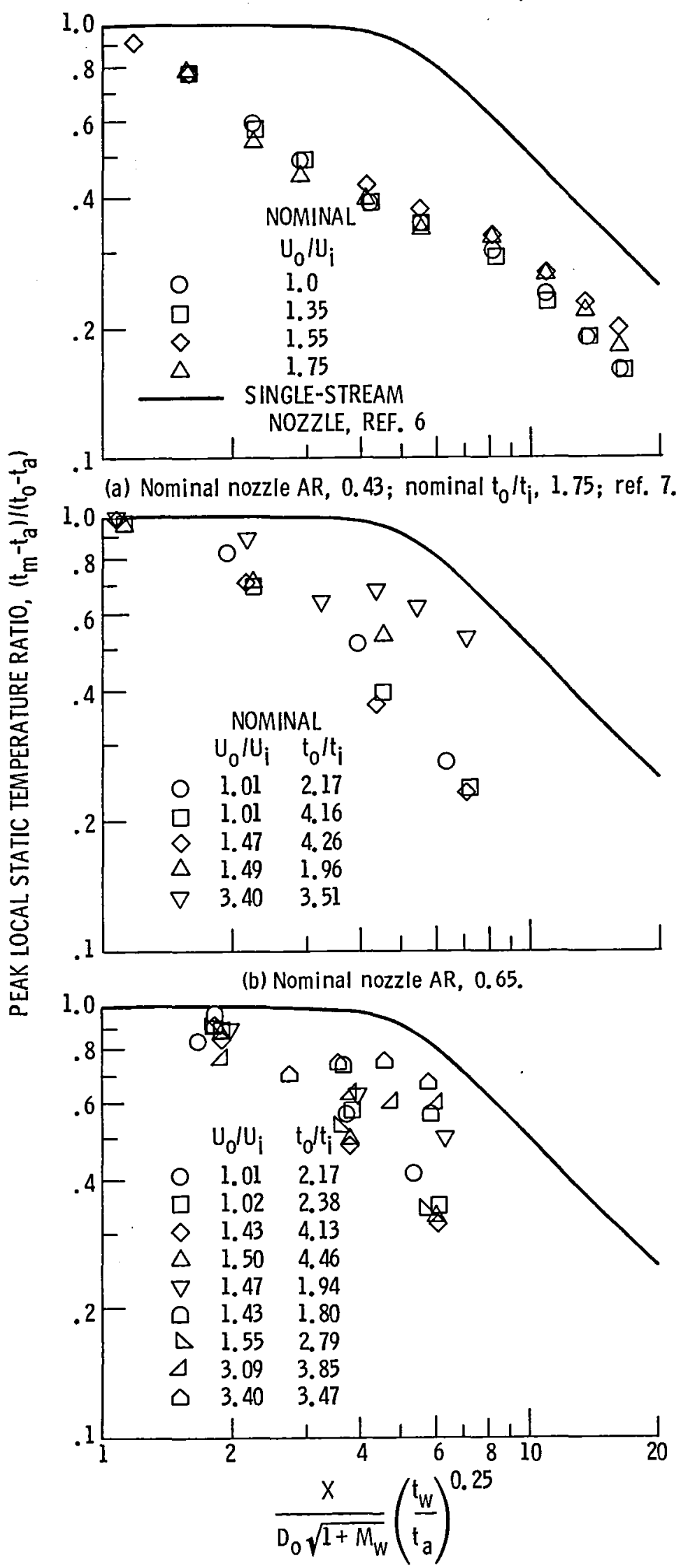

(c) Nominal nozzle AR, 0.76 .

Figure 10. - Peak two-stream inverted-profile static temperature decay data. 


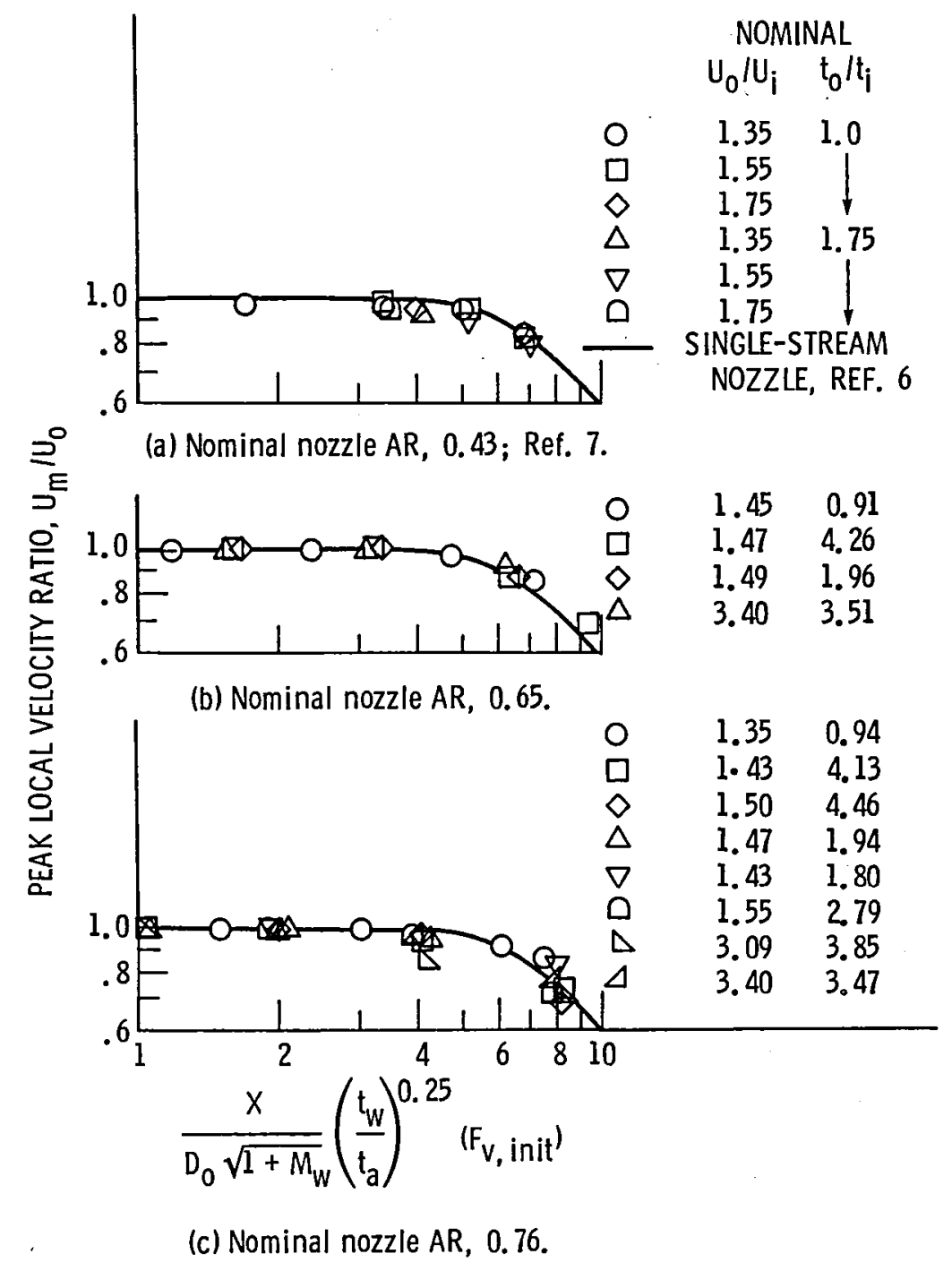

Figure 11. - Correlated peak velocity decay in the initial mixing region. 


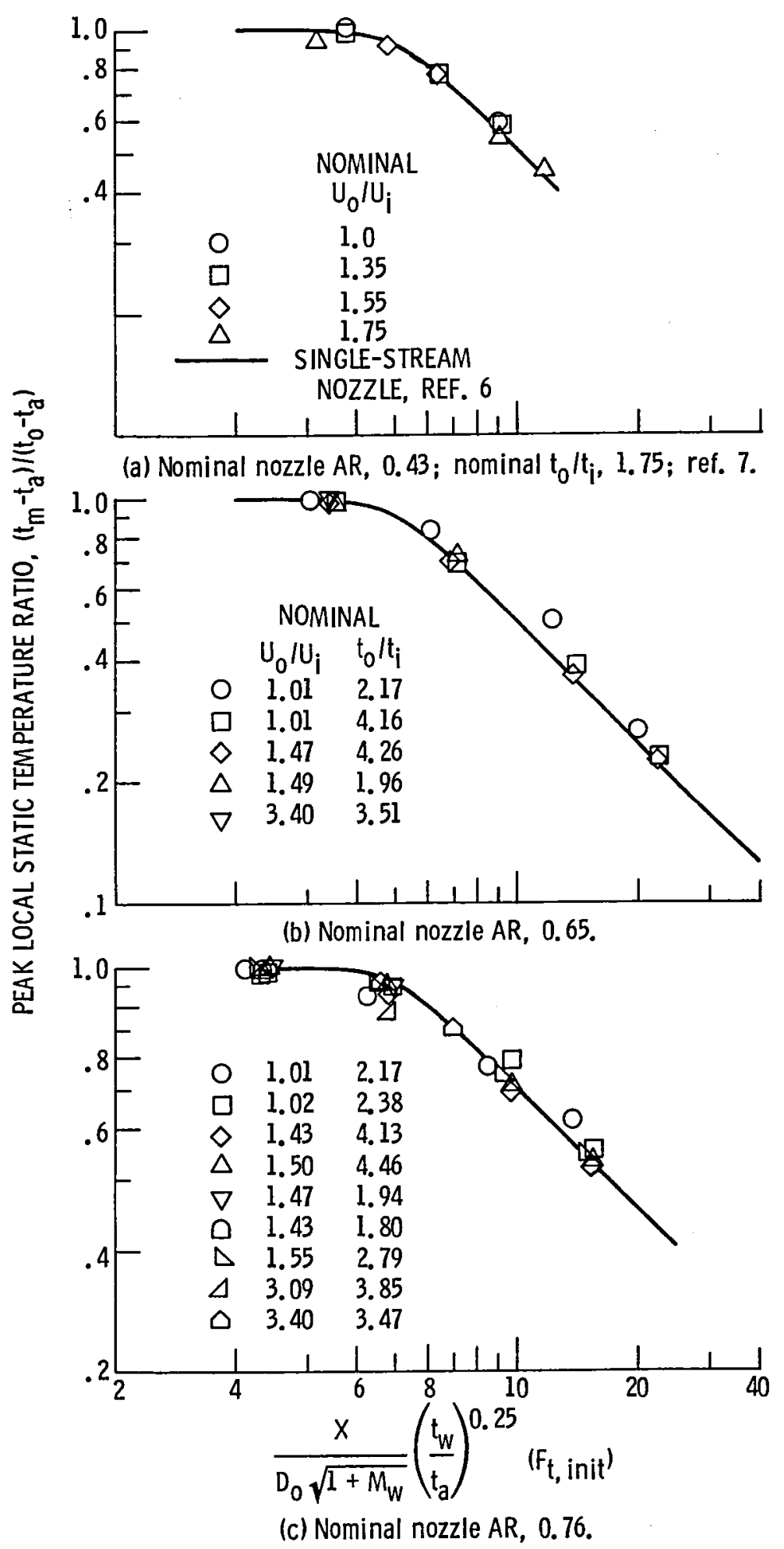

Figure 12. - Correlated peak static temperature decay in the initial mixing region. 


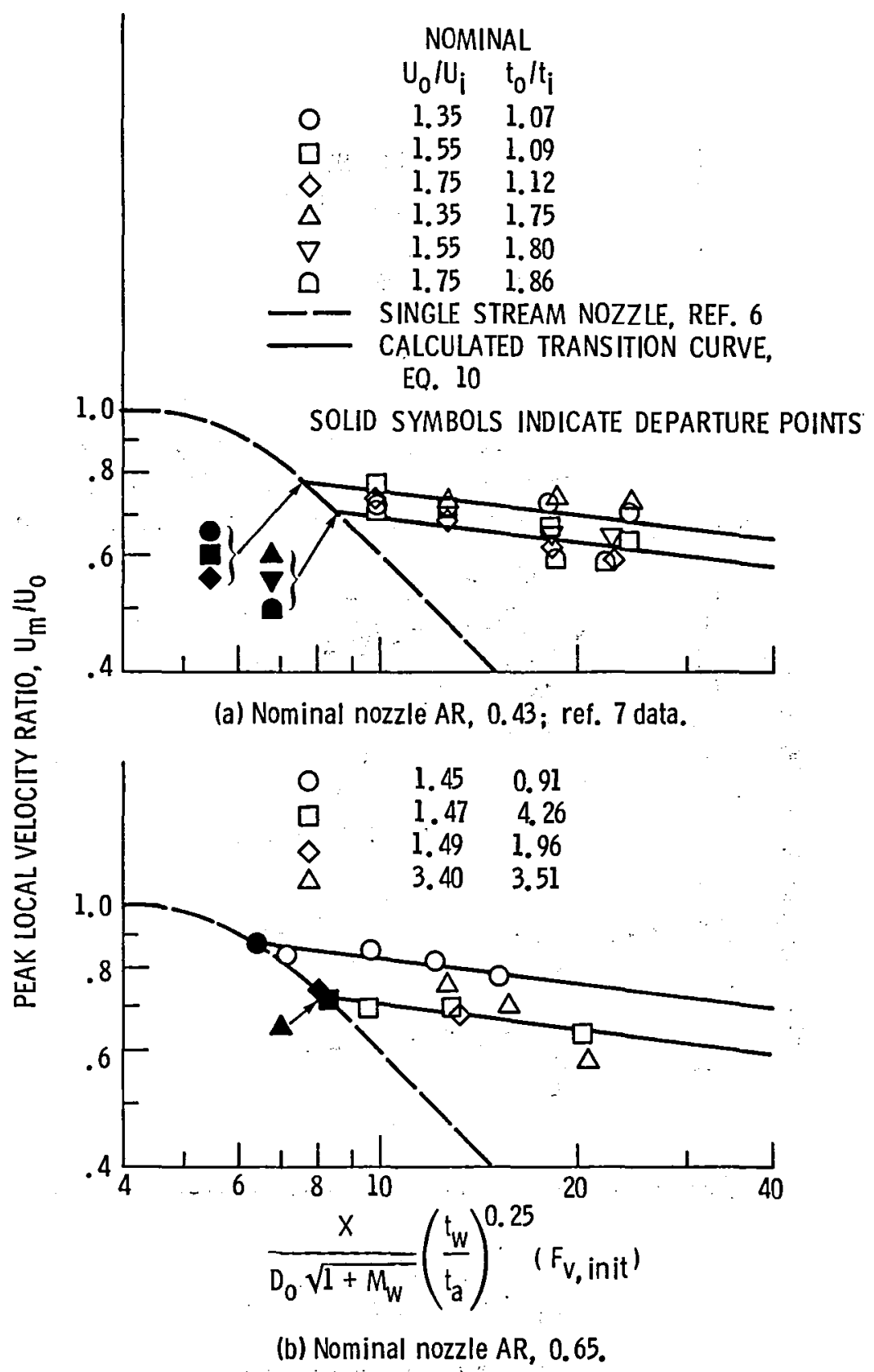

Figure 13. - Correlated peak velocity decay in the transition region. 


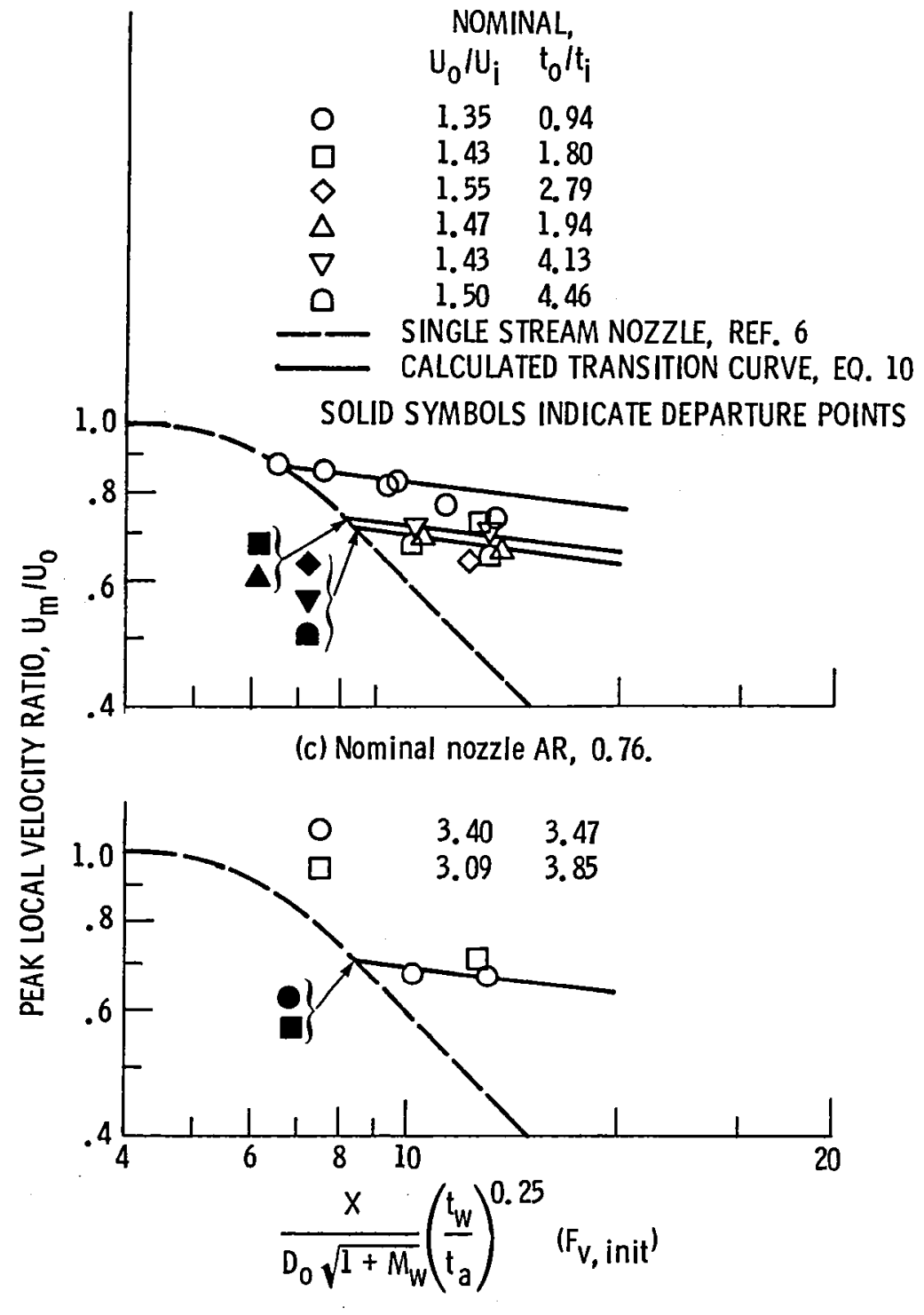

(d) Nominal nozzle AR, 0.76 .

Figure 13. - Concluded. 


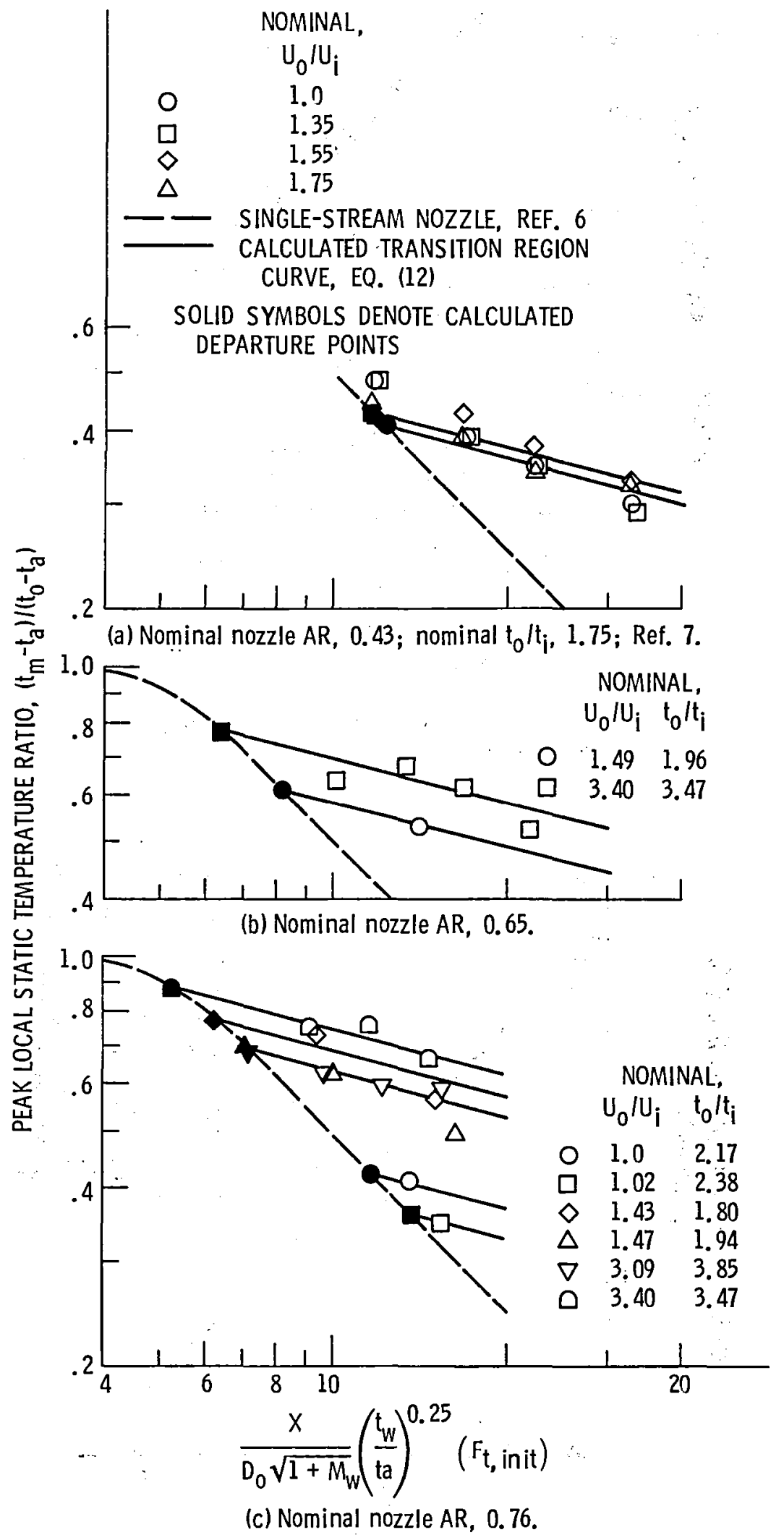

Figure 14. - Correlated peak static temperature decay in transition region. 


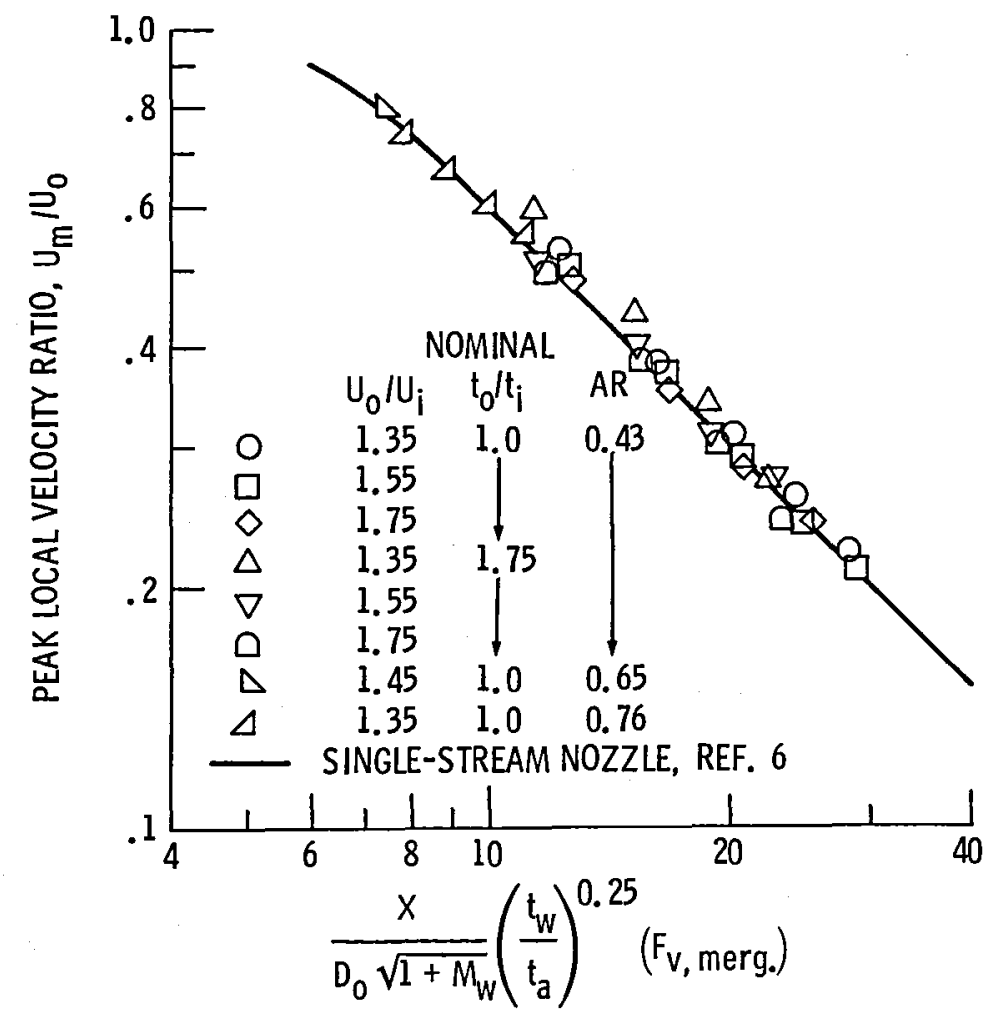

Figure 15. - Correlated peak velocity decay in fully merged flow region.

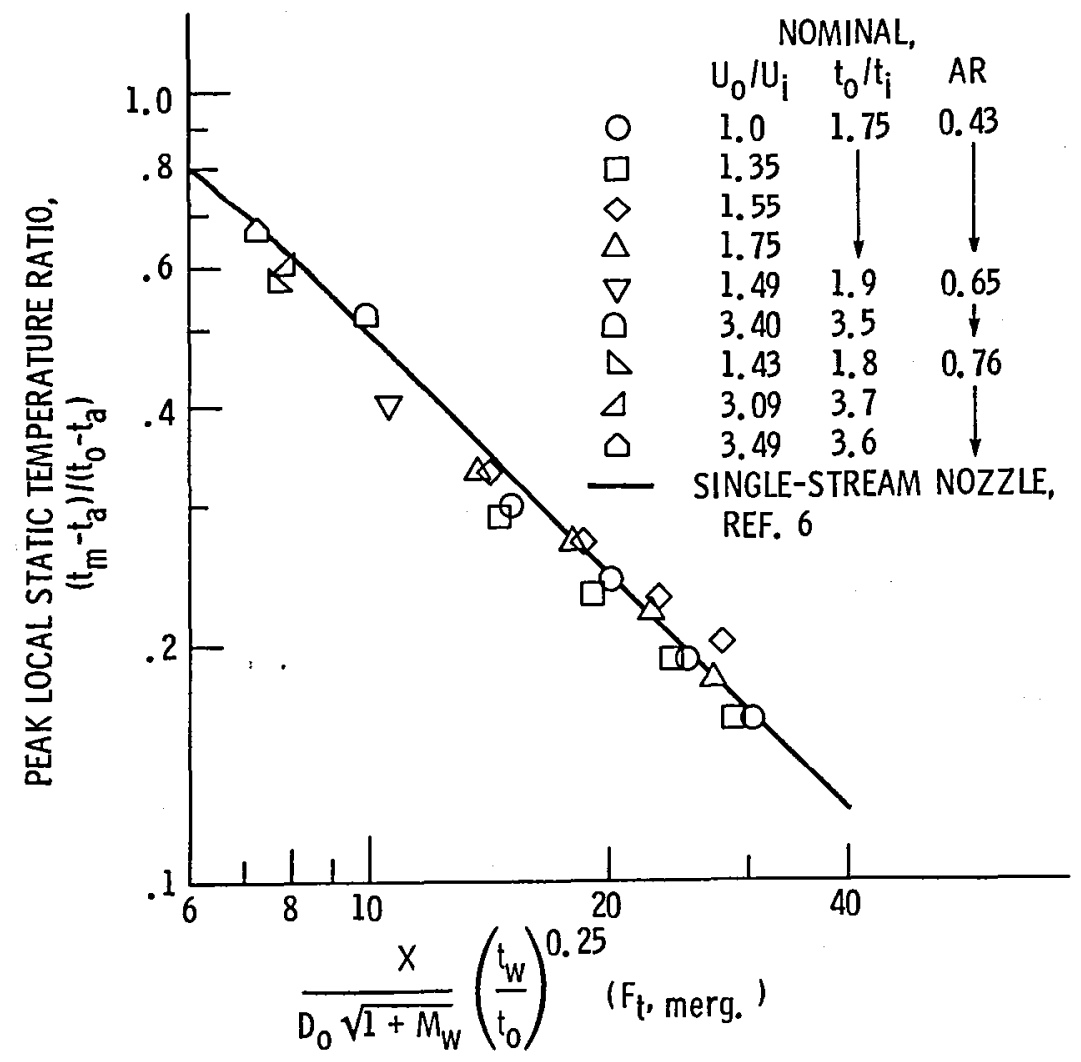

Figure 16. - Correlated peak static temperature decay in fully merged flow region. 

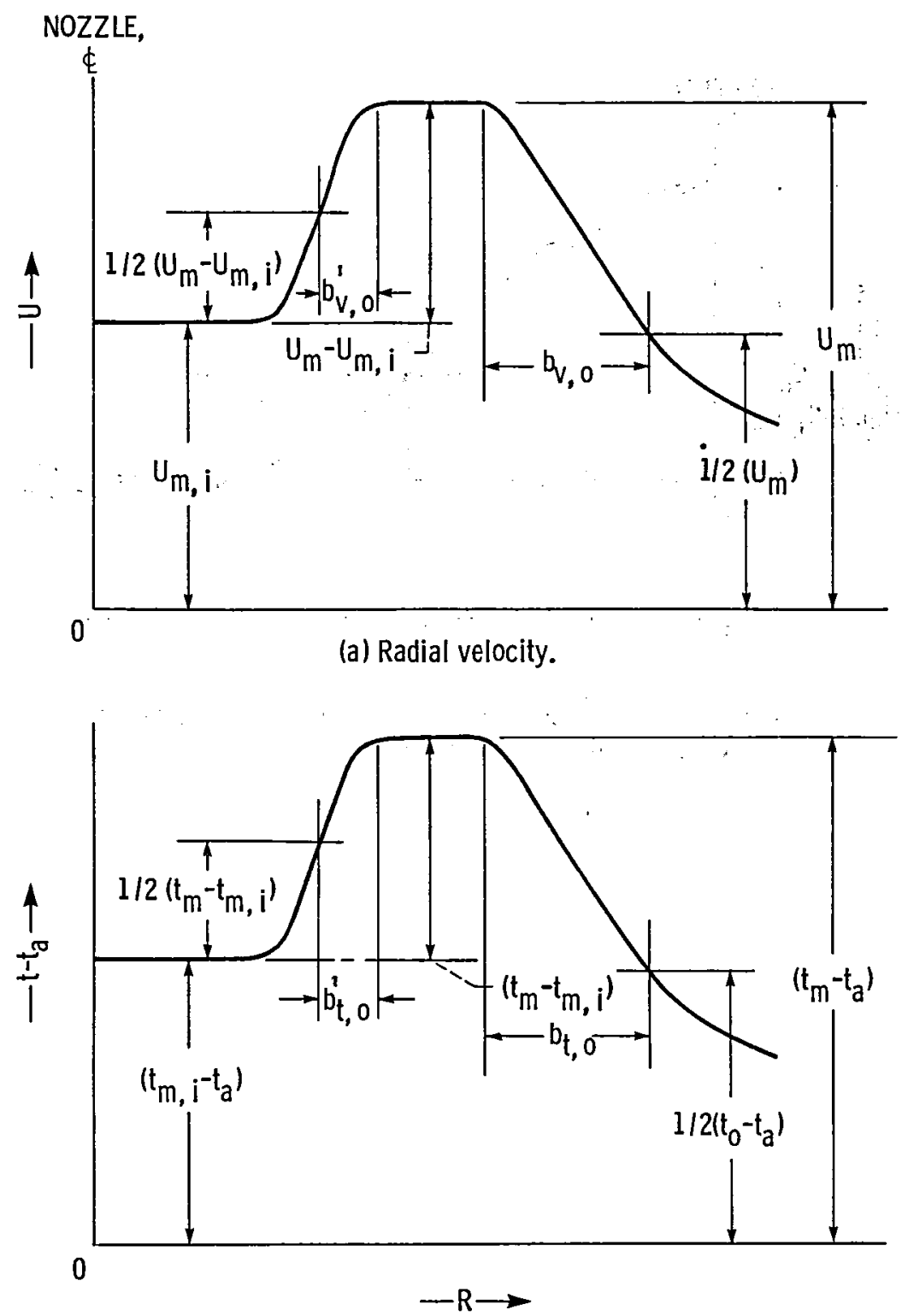

(b) Radial temperature.

Figure 17. - Pertinent variables for radial velocity/temperature profiles in initial mixing region. 


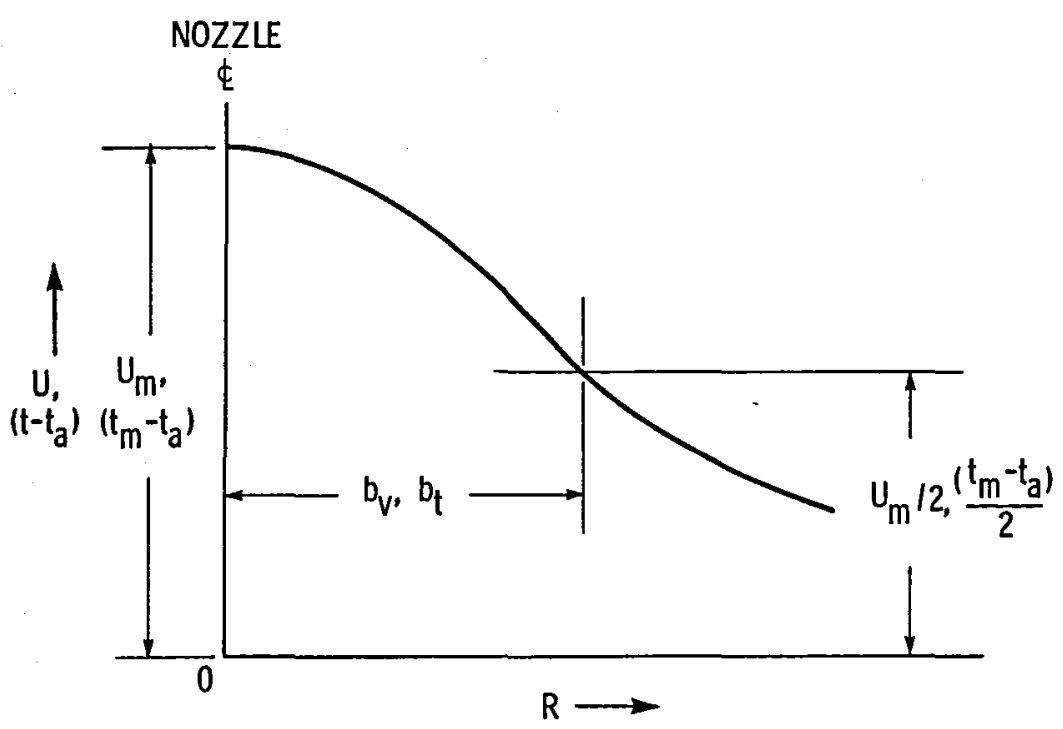

Figure 18. - Pertinent variables for radial velocity/temperature profiles in fully merged flow region. 


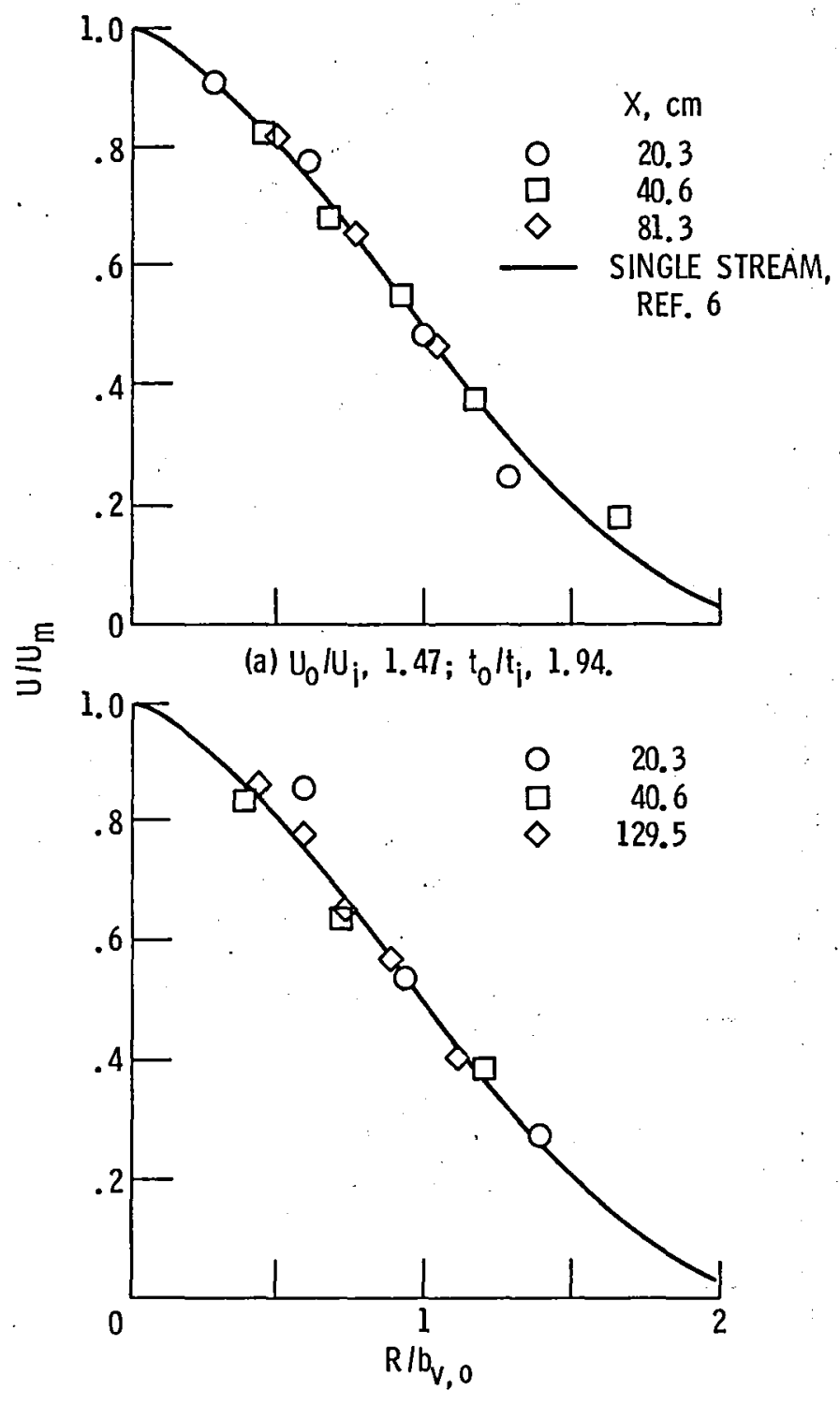

(b) $U_{0} / U_{i}, 1.43 ; t_{0} / t_{i}, 4.13$.

Figure 19. - Typical correlation of two-stream radial velocity profile decay. Nominal nozzle AR, 0.76 . 


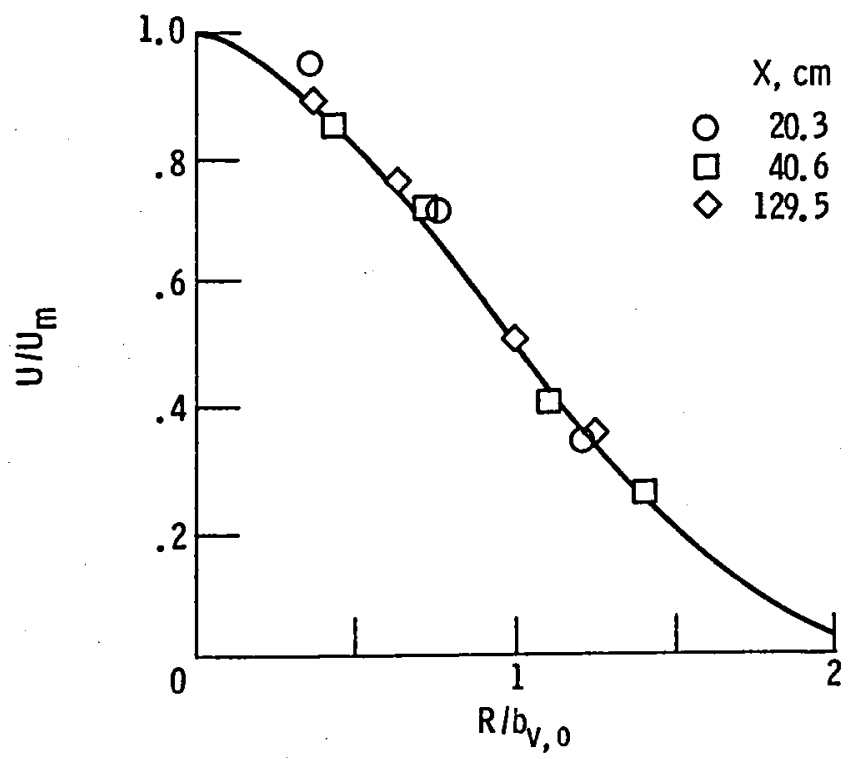

(c) $U_{0} / U_{i}, 3.40 ; t_{0} / t_{i}, 3.46$.

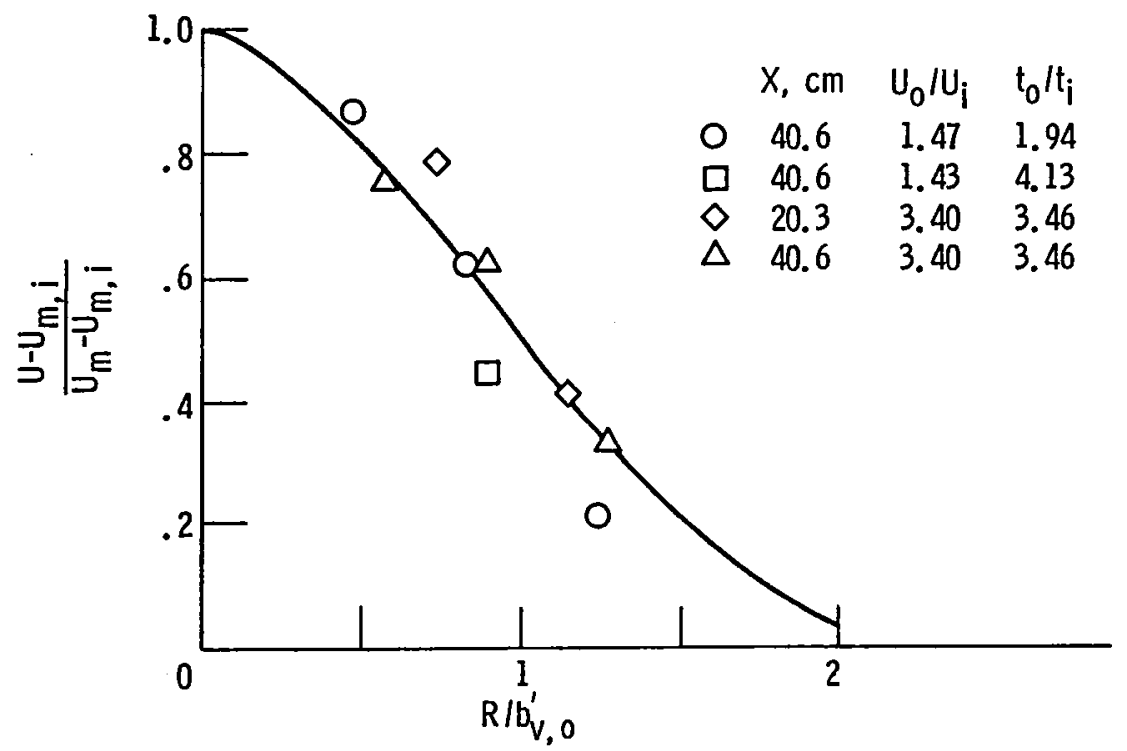

(d) Various flow conditions.

Figure 19. - Concluded. 


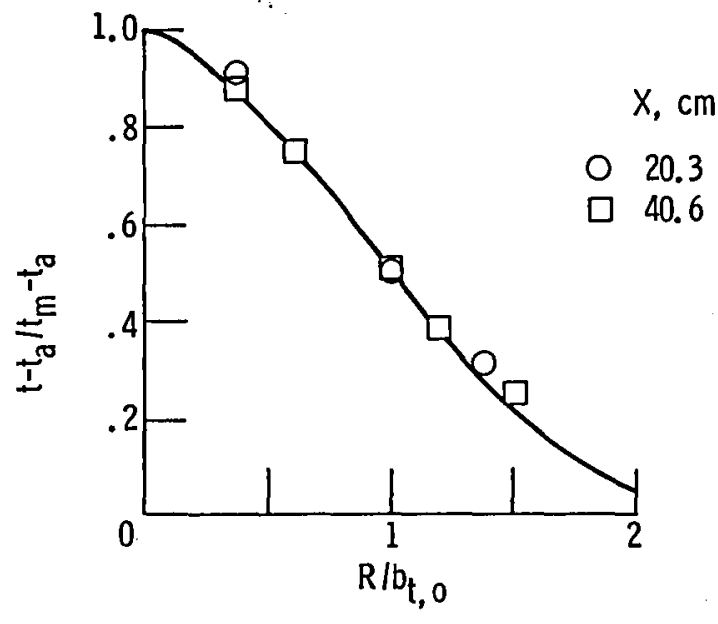

(c) $U_{0} / U_{i}, 3.41 ; t_{0} / t_{i}, 3.46$.

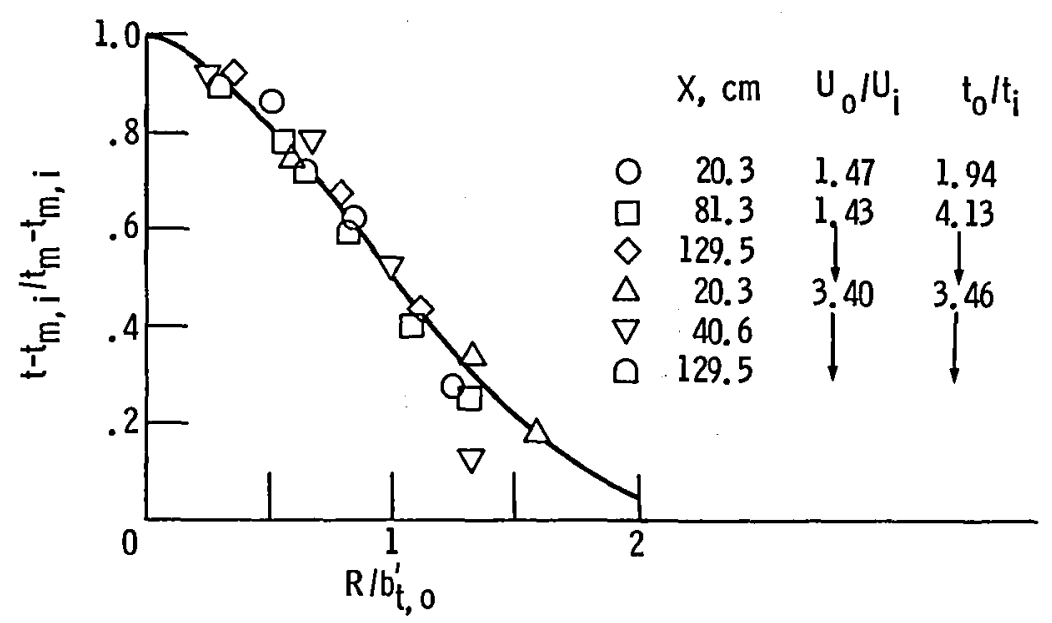

(d) Various flow conditions.

Figure 20. - Typical correlation of two-stream radial static temperature decay, nominal nozzle AR, 0.76 .

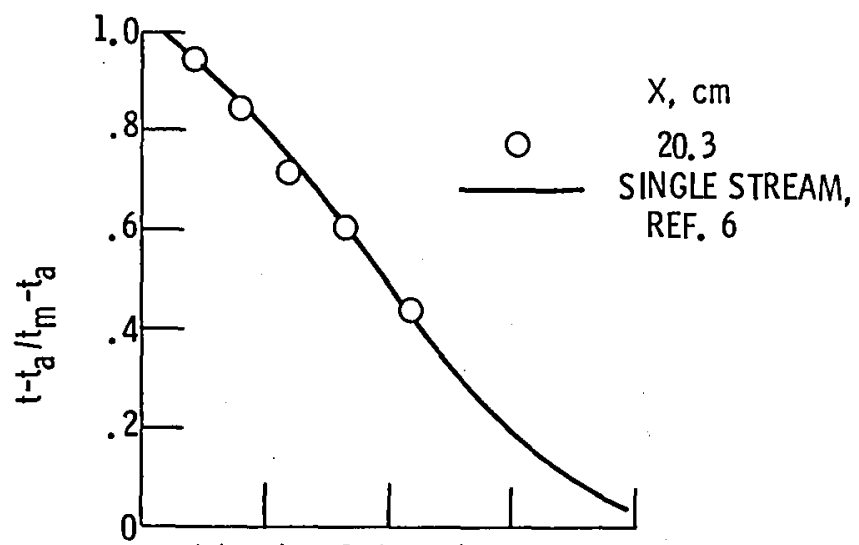

(a) $U_{0} / U_{i}, 1.47 ; t_{0} / t_{i}, 1.94$.

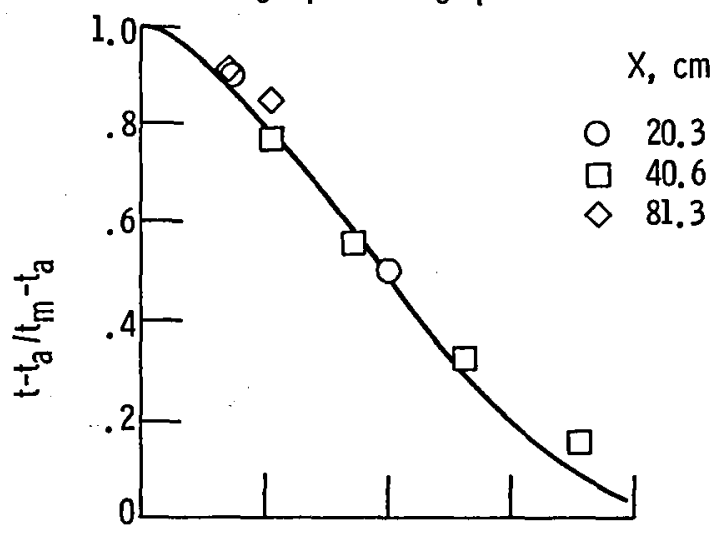

(b) $U_{0} / U_{i}, 1.43 ; t_{0} / t_{j}, 4.13$. 


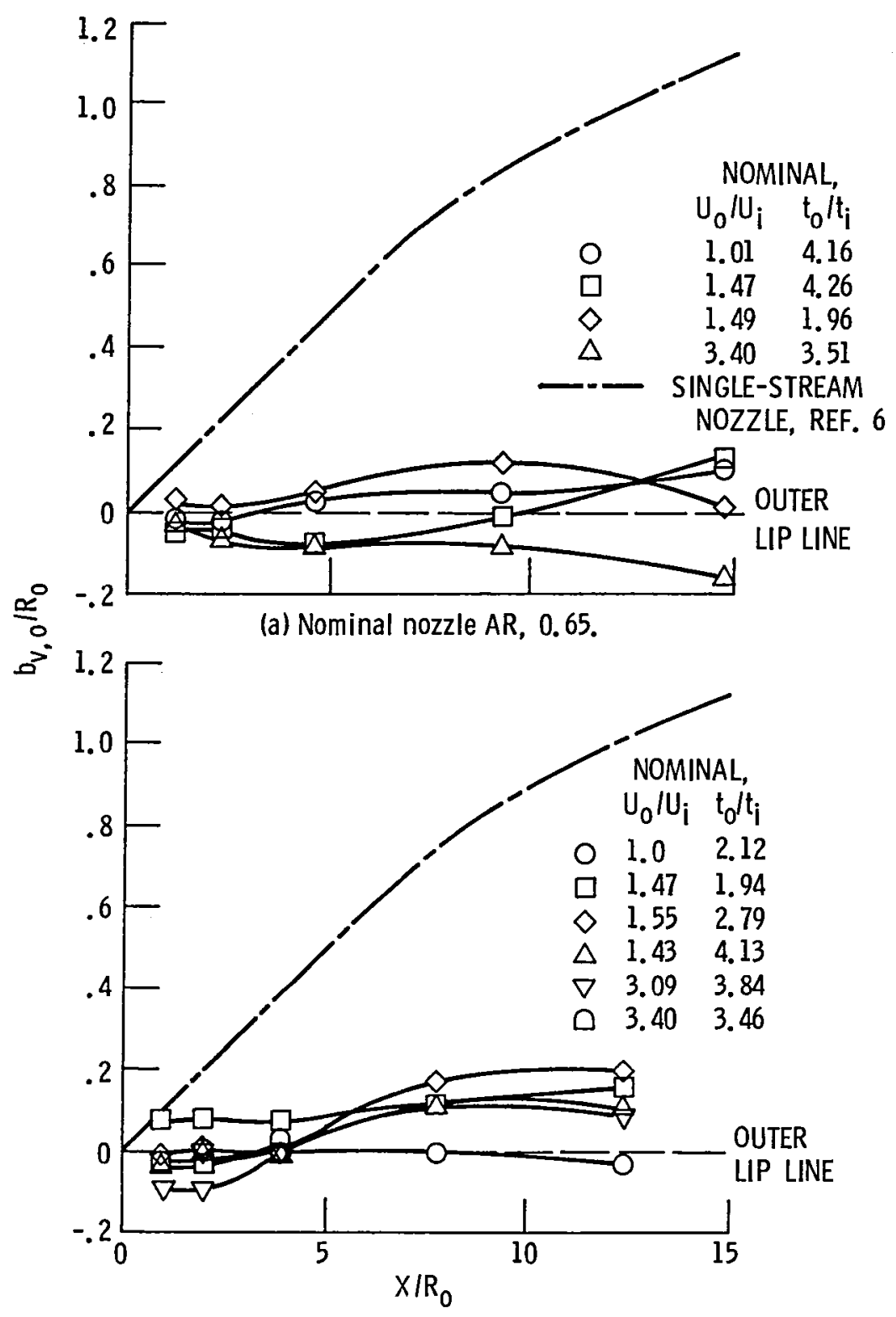

(b) Nominal nozzle AR, 0.76 .

Figure 21. - Velocity half-width on outer stream ambient side. 


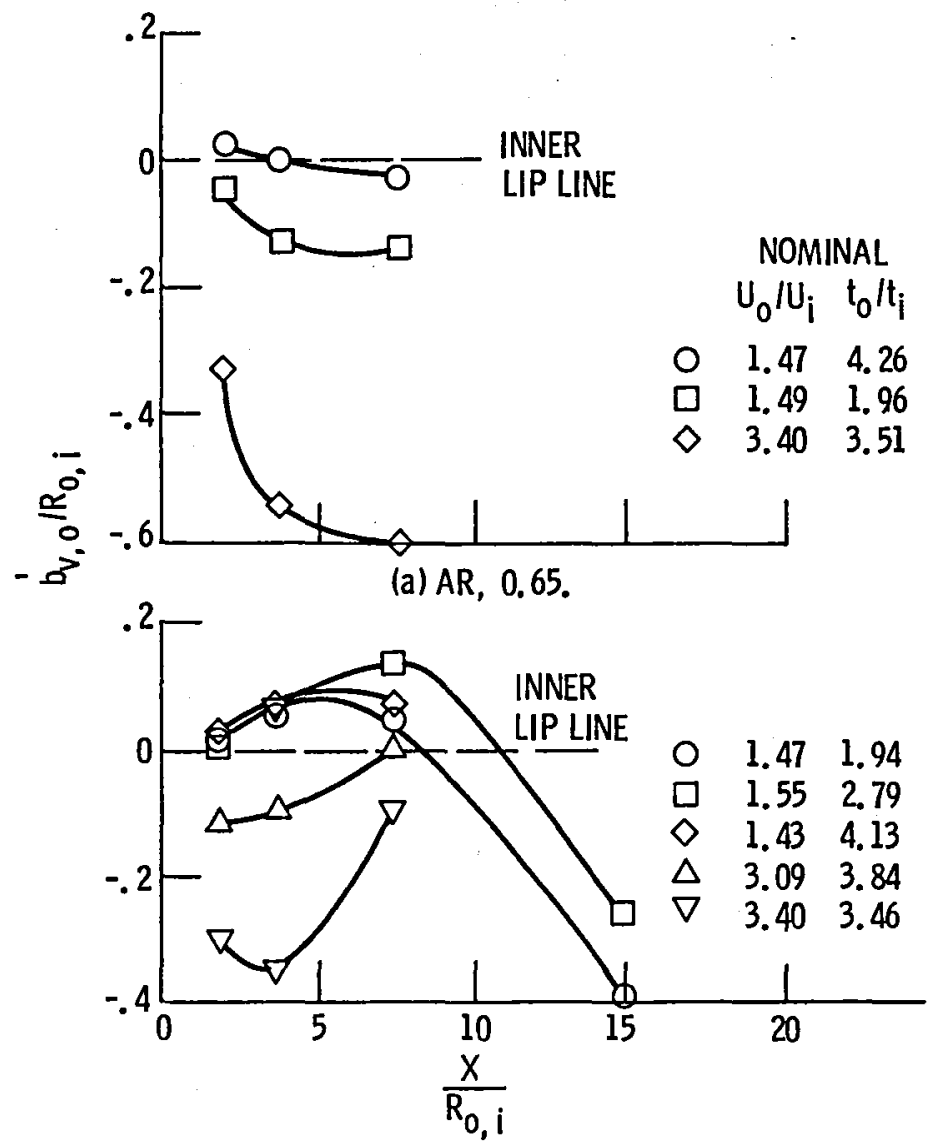

(b) AR, 0.76 .

Figure 22. - Velocity half-width on inner side of outer stream. 


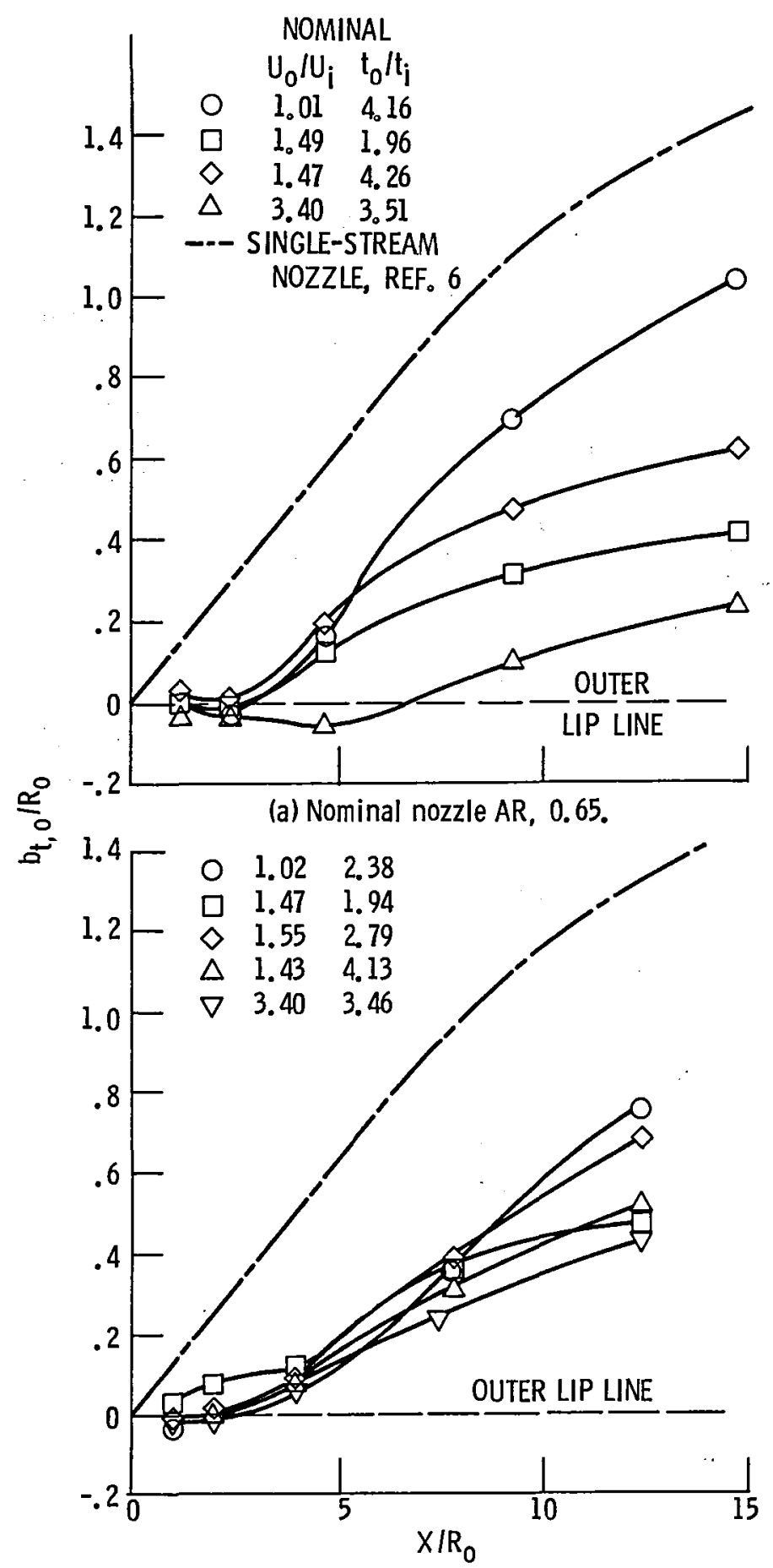

(b) Nominal nozzle AR, 0.76 .

Figure 23. - Static temperature half-width on outer stream ambient side. 


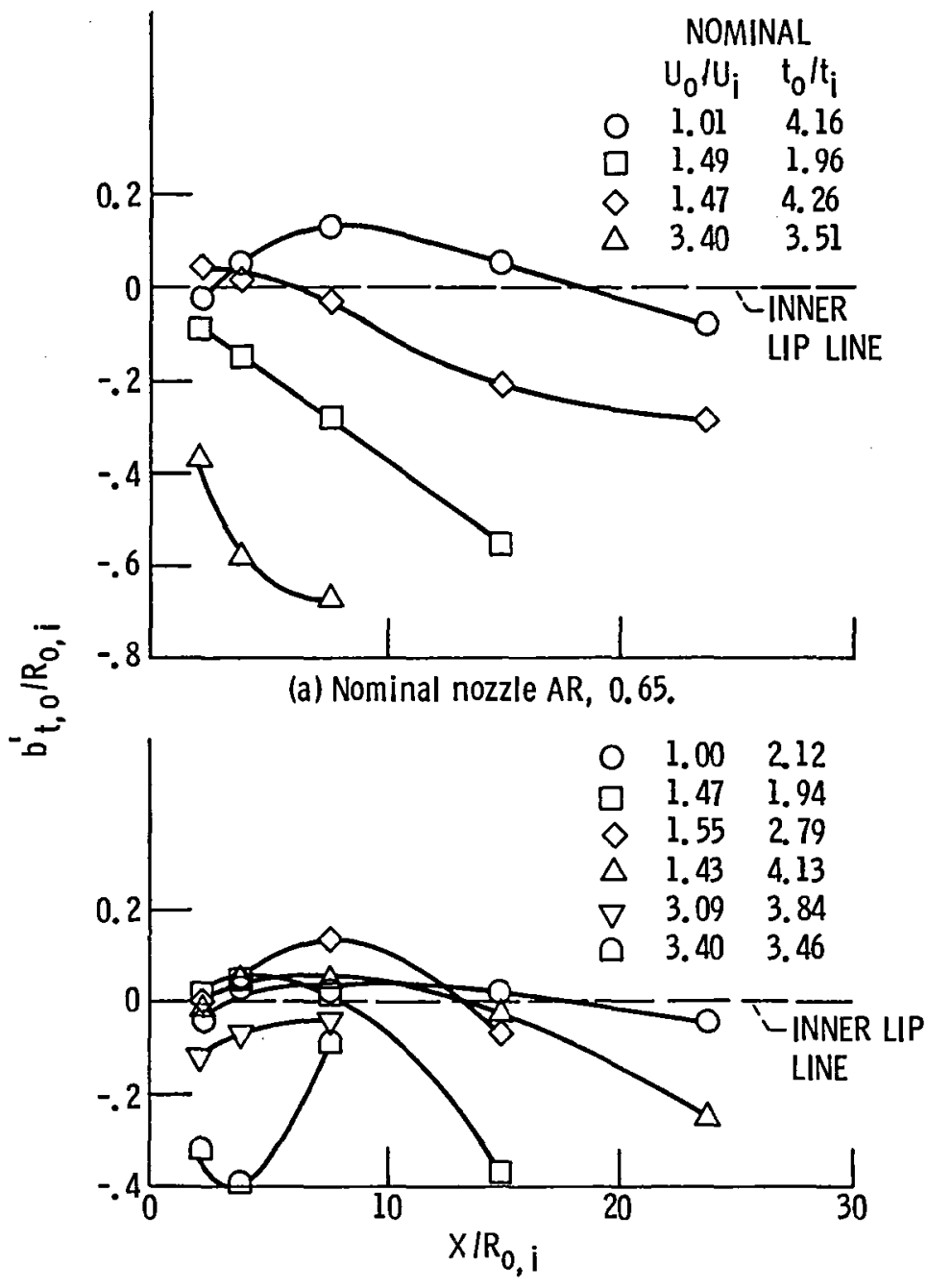

(b) Nominal nozzle AR, 0.76

Figure 24. - Static temperature half-width on inner side of outer stream. 


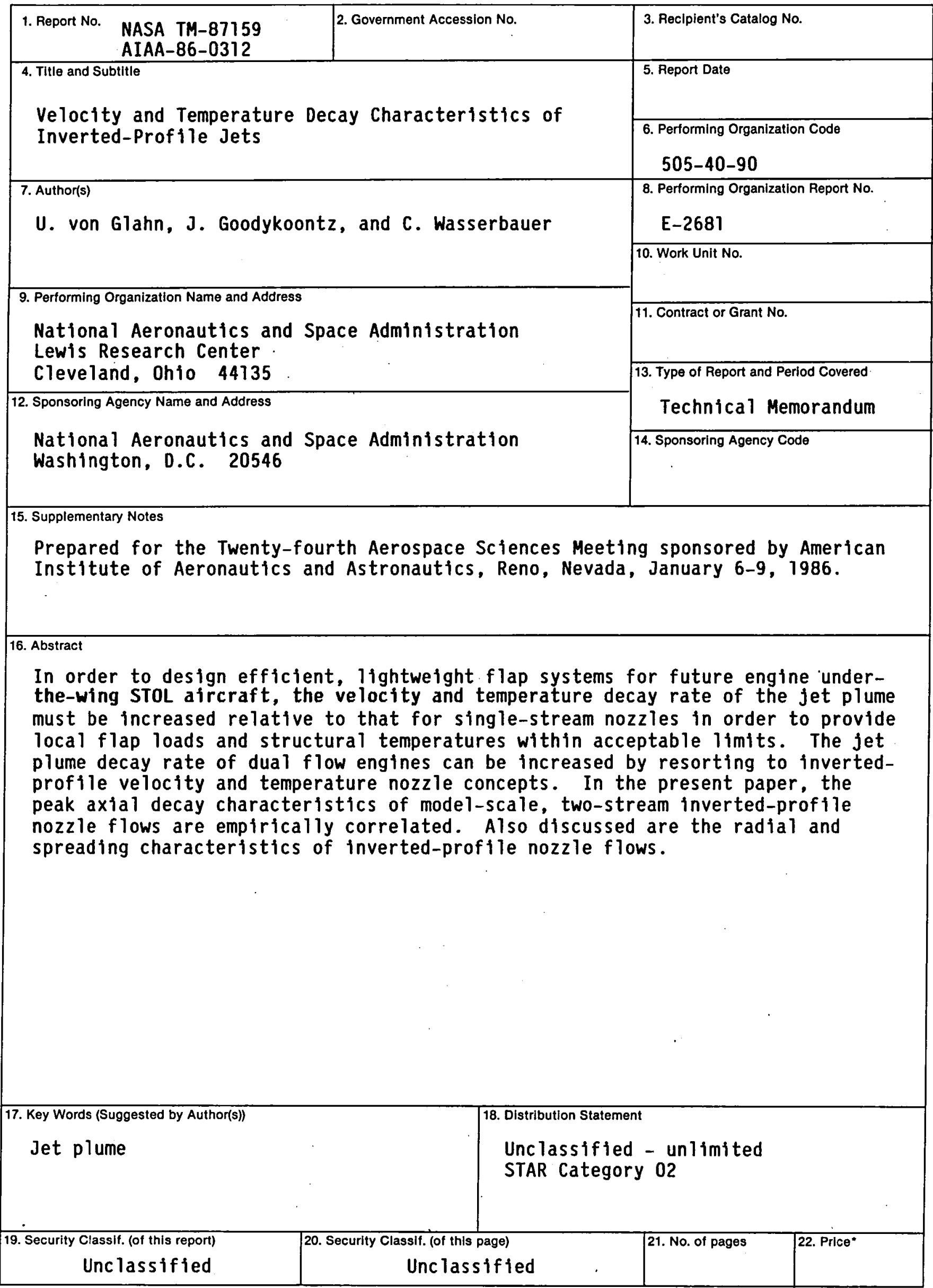

*For sale by the National Technical Information Service, Springfield, Virginia 22161 


\section{End of Document}

